\title{
Diameter effect on the heat transfer of supercritical hydrocarbon fuel in horizontal tubes under turbulent conditions
}

\author{
Zeyuan Cheng ${ }^{\mathrm{a}}$, Zhi Tao ${ }^{\mathrm{a}}$, Jianqin Zhu ${ }^{\mathrm{a}, *}$, Hongwei $\mathrm{Wu}^{\mathrm{b}, * *}$ \\ a National Key Laboratory of Science and Technology on Aeroengine Aerothermodynamics, \\ School of Energy and Power Engineering, Beihang University, Beijing, 100191, P.R. China

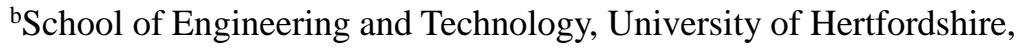 \\ Hatfield, AL10 9AB, United Kingdom \\ * Corresponding author. Email: zhujianqinbuaa@ sina.com Tel. +86(010)82339181 \\ ${ }^{* * *}$ Corresponding author. Email: h.wu6@herts.ac.uk Tel. +44(0)1707 284265; \\ Fax. +44(0)1707 285086
}

\begin{abstract}
This article presented a numerical investigation of supercritical heat transfer of hydrocarbon fuel in a series of horizontal tubes with different diameters. It has been carried out by solving Reynolds averaging equations of mass, momentum and energy with the LS low-Reynolds number turbulence model using the pressure-based segregated solver based on the finite volume method. For the purpose of comparison, a four-species surrogate model and a ten-species surrogate model of aviation kerosene RP-3 (Rocket Propellant 3) were tested against the published experimental data. In the current study, tube diameter varied from $2 \mathrm{~mm}$ to $10 \mathrm{~mm}$ and pressure was $3 \mathrm{MPa}$ with heat flux to mass flux ratios ranging from 0.25 to $0.71 \mathrm{~kJ} / \mathrm{kg}$. It has been found that the buoyancy has significant effect on wall temperature non-uniformity in the horizontal tube. With the increase of diameter, the buoyancy effect enhances and the thermal-induced acceleration effect reduces. The buoyancy effect makes wall temperature at the top and bottom generatrices of horizontal tube increase and decrease, respectively. Due to the coupled effect of buoyancy and thermal-induced acceleration caused by the significant change of the properties, as diameter increases, heat transfer deteriorates dramatically at the top generatrix but remains almost unchanged at the bottom generatrix at high heat flux to mass flux ratio. Heat transfer enhancement is observed at low heat flux to mass flux ratio when tube diameter is less than $6 \mathrm{~mm}$. Moreover, the safety analysis has been performed in order to optimally design the supercritical cooling system.
\end{abstract}

Keywords: Supercritical; Hydrocarbon; Heat transfer; Diameter; Horizontal tube. 


\begin{tabular}{|c|c|c|c|}
\hline \multicolumn{4}{|c|}{ Nomenclature } \\
\hline$\overline{a_{n}}$ & Coefficients in MBWR equation & $R e_{t}$ & Turbulent Reynolds number \\
\hline$A$ & Integral area & $S_{k}$ & Source term in $k$ equation \\
\hline$B o^{*}$ & Buoyancy parameter & $S_{\varepsilon}$ & Source term in $\varepsilon$ equation \\
\hline$C_{P}$ & $\begin{array}{l}\text { Specific heat at constant pressure } \\
\left(\mathrm{J} \cdot \mathrm{kg}^{-1} \cdot \mathrm{K}^{-1}\right)\end{array}$ & $T$ & Temperature (K) \\
\hline$C_{V}$ & $\begin{array}{l}\text { Specific heat at constant volume } \\
\left(\mathrm{J} \cdot \mathrm{kg}^{-1} \cdot \mathrm{K}^{-1}\right)\end{array}$ & $u$ & Velocity $\left(\mathrm{m} \cdot \mathrm{s}^{-1}\right)$ \\
\hline$C_{\varepsilon 1}, C_{\varepsilon 2}$ & Constants in $\varepsilon$ equation & $x$ & Axial coordinate $(\mathrm{mm})$ \\
\hline$C_{\mu}$ & Constant in eddy viscosity & $y$ & Radial coordinate (mm) \\
\hline$d$ & Inner diameter of tube (mm) & $y+$ & Wall non-dimensional distance \\
\hline$D$ & Outer diameter of tube (mm) & & \\
\hline$f_{1}, f_{2}$ & Functions in $\varepsilon$ equation & \multicolumn{2}{|c|}{ Greek symbols } \\
\hline$f_{\mu}$ & Damping function & $\beta$ & Thermal expansion coefficient $\left(\mathrm{K}^{-1}\right)$ \\
\hline$F_{p}^{0}, F_{Q}{ }^{0}$ & Correction factor, $\approx 1$ & $\gamma$ & $1 /\left(\rho_{c 0}\right)^{2}$ \\
\hline$g$ & Gravitational acceleration $\left(\mathrm{m} \cdot \mathrm{s}^{-2}\right)$ & $\varepsilon$ & Dissipation rate of $k\left(\mathrm{~m}^{-2} \cdot \mathrm{s}^{-3}\right)$ \\
\hline$G$ & Mass flux $\left(\mathrm{kg} \cdot \mathrm{m}^{-2} \cdot \mathrm{s}^{-1}\right)$ & $\lambda$ & Thermal conductivity $\left(\mathrm{W} \cdot \mathrm{m}^{-1} \cdot \mathrm{K}^{-1}\right)$ \\
\hline$G_{k}$ & $\begin{array}{l}\text { Turbulence production by buoyancy } \\
\left(\mathrm{kg} \cdot \mathrm{m}^{-1} \cdot \mathrm{s}^{-3}\right)\end{array}$ & $\mu$ & Molecular viscosity $(\mu \mathrm{Pa} \cdot \mathrm{s})$ \\
\hline$G r^{*}$ & Grashof number & $\mu_{t}$ & Turbulent viscosity $(\mu \mathrm{Pa} \cdot \mathrm{s})$ \\
\hline$h$ & Heat transfer coefficient & $\rho$ & Density $\left(\mathrm{kg} \cdot \mathrm{m}^{-3}\right)$ \\
\hline$H$ & Enthalpy $\left(\mathrm{J} \cdot \mathrm{kg}^{-1}\right)$ & $\sigma_{k}, \sigma_{\epsilon}$ & Turbulent Prandtl number \\
\hline$k$ & Turbulent kinetic energy $\left(\mathrm{m}^{-2} \cdot \mathrm{s}^{-2}\right)$ & $\tau$ & Shear stress $\left(\mathrm{kg} \cdot \mathrm{m}^{-1} \cdot \mathrm{s}^{-2}\right)$ \\
\hline$K_{v}$ & Flow acceleration parameter & $v$ & Kinematic viscosity, $v=\mu l \rho$ \\
\hline$L$ & Total length of tube (mm) & & \\
\hline$M$ & Molar mass & \multicolumn{2}{|c|}{ Subscripts/Superscripts } \\
\hline$N u$ & Nusselt number & 0 & Reference substance \\
\hline$P$ & Pressure (Mpa) & $b$ & Bulk \\
\hline $\operatorname{Pr}$ & Molecular Prandtl number & $c$ & Critical \\
\hline$P r_{t}$ & Turbulent Prandtl number & in & Inlet \\
\hline$q$ & Wall heat flux $\left(\mathrm{kW} \cdot \mathrm{m}^{-2}\right)$ & $r$ & Relative \\
\hline$R$ & Radius (mm) & $w$ & Wall \\
\hline $\operatorname{Re}$ & Reynolds number & $l$ & Superscript for low pressure \\
\hline
\end{tabular}




\section{Introduction}

Scramjet engine has been widely accepted as one of the most promising power systems of hypersonic vehicles due to its high efficiency and high thrust-weight ratio when cruising at 6-10 Mach. However, high speed cruise brings the significant friction heat to the Scramjet, leading to the severe thermal management problem. In order to overcome this problem, the regenerative cooling technique is necessary to ensure the engine working reliably [1]. As a potential coolant, hydrocarbon fuel under supercritical pressure absorbs heat from combustor when the unburned coolant is flowing through cooling channel. Afterwards, the absorbed heat is returned to the work cycle when the coolant is injected into the combustor. During the cooling process, hydrocarbon fuel undergoes the transcritical process, that is, its temperature increases from subcritical temperature to supercritical temperature due to the non-symmetric heating and then it changes into the supercritical fluid. Similar cooling process with symmetric heating occurs in CCA (cool cooling air) technology in the modern advanced gas turbine engines [2]. The fascinating feature of the fluid under supercritical pressure is that the thermophysical property varies significantly with temperature in the vicinity of pseudo-critical point. Therefore, to investigate the characteristics of supercritical heat transfer is becoming one of the most challenging issues and an important subject [3].

Cagniard de la Tour in 1822 [4] was perhaps the first one to study the supercritical phenomenon and then the research on flow and heat transfer under supercritical pressure has never been stopped [5,6]. With the development of study, some detailed reviews of heat transfer at supercritical pressure have been provided [7-9]. It is believed that at low heat flux to mass flux ratio, heat transfer enhancement characterized by the gently changing temperature and high heat transfer coefficient near pseudo-critical point occurs; while heat transfer deterioration featured by the suddenly raised temperature occurs at high heat flux [10]. Generally, due to high heat sink capacity, heat transfer enhancement is preferred in the practical applications, and to avoid the material failure caused by sufficient high wall temperature, more attention is paid to reduce heat transfer deterioration [11].

In both heat transfer enhancement and heat transfer deterioration modes, supercritical heat transfer is influenced by the variable property effect, coupled with the buoyancy effect and thermal-induced acceleration effect. The effect of buoyancy and thermal-induced acceleration on supercritical heat transfer may vary when flow direction changes from the vertical flow to the horizontal flow. $\mathrm{Li}$ et al. [12] experimentally studied the distinctions of heat transfer characteristics between horizontal and vertical upward flows of supercritical pressure water. It has been found that at high heat flux to mass flux ratio, the thermal-induced acceleration is the key factor leading to heat transfer deterioration in the vertical flow. Whereas in the horizontal flow, the buoyancy effect played a larger effect than in the vertical flow. From the numerical simulation of Li et al. [13], it was stated that as heat transfer deterioration happened in the horizontal tube, the buoyancy effect resulted in the vertical stratification and the accumulation of the light supercritical pressure fluid. More deeply, the buoyancy effect was proved to be responsible for the occurrence of the secondary flows and the circumferential nonuniformity on heat transfer in the horizontal flow of supercritical water [14].

Except of the flow direction, there are other factors, like diameter, which may have significant effect on supercritical heat transfer. Shang et al. [15] numerically investigated the diameter effect to heat transfer of supercritical water. It has been found to be easier to induce heat 
transfer deterioration in large diameter tube $(10 \mathrm{~mm})$, and heat transfer deterioration gradually weakens with mass flux increasing. Afterwards, the experiment of water flowing in horizontal tubes at supercritical pressure has been carried out by Yu et al. [16]. It has been concluded that the diameter has very little effect on heat transfer at low heat flux to mass flux ratio $(0.16 \mathrm{~kJ} / \mathrm{kg})$, but can result in the obvious buoyancy effect at high heat flux to mass flux ratio $(0.4 \mathrm{~kJ} / \mathrm{kg})$. Yildiz [17] provided a detailed survey about the tube diameter effect on heat transfer at supercritical pressures and found that the large diameter might contribute to the reduced heat transfer coefficient in heat transfer deterioration mode. The latest study on the hydraulic characteristics of supercritical hydrocarbon fuel in tubes of different diameters was experimentally investigated by Guo et al. [18], and the magnitude of pressure drop along the tube was found to be much larger in the larger diameter tube.

All the studies mentioned above have shown significant results concerning the diameter effect and flow direction effect on supercritical flow, but the comprehensive study combining the diameter effect with the horizontal flow is less and the existed research is mainly focused on water and carbon dioxide [19-22]. It has been concluded that the heat transfer characteristics of hydrocarbon fuel at supercritical pressure are quite complicated and the understanding of heat transfer mechanism including heat transfer deterioration and heat transfer enhancement has been far from complete. Actually, the horizontal flow of supercritical hydrocarbon fuel in the cooling process is common during the flight of the Scramjet, and the further study in this regard is urgently needed especially at various tube diameters to develop more practical methods to better understand the heat transfer behavior of hydrocarbon fuel at supercritical pressure. In order to fill in the research gap in this field, a four-species surrogate model and ten-species surrogate model of aviation kerosene RP-3 (Rocket Propellant 3) with LS low-Reynolds number turbulence model were proposed and tested against the published experimental data. Finally, the diameter effect on supercritical heat transfer of hydrocarbon fuel in a series of horizontal tubes was investigated numerically. The nonuniform heat transfer characteristics along the circumference along with buoyancy effect and thermal-induced acceleration effect have been analyzed to better understand and explain the heat transfer behavior at high and low heat flux to mass flux ratios, paying attention to heat transfer deterioration of the horizontal flow at various diameters.

\section{Thermophysical properties of hydrocarbon fuel}

It is well known that there are multiple types of hydrocarbon fuels used in aero vehicles, i.e., Jet-A, JP-6, JP-7 and JP-8. In the present study, RP-3, a representative aviation kerosene used in the Scramjet, is selected. It is composed of different fractions of hydrocarbon compounds, and Table 1 lists the main components of RP-3 obtained by gas chromatography - mass spectrometry.

Table 1 Main components of RP-3.

\begin{tabular}{ccc}
\hline No. & Component & Mass fractions/\% \\
\hline 1 & Alkane & 52.44 \\
2 & Benzene & 18.53 \\
3 & Cyclane & 15.54 \\
4 & Alkene & 7.64 \\
5 & Naphthalene & 4.39 \\
6 & Other & 1.46 \\
\hline
\end{tabular}


It is noted that there are hundreds of complex species in RP-3 and its composition may change with the production batch. Therefore, it is a common practice to use the surrogate fuel [23] to simulate the thermophysical properties of RP-3, rather than studying each of these components one by one. Two widely used surrogate models, four-species surrogate model [24] and ten-species surrogate model [25], will be considered for the purpose of comparison, as shown in Table 2.

Table 2 Two kinds of surrogate models for RP-3 and real fuel.

\begin{tabular}{|c|c|c|}
\hline Model & Composition(molar fraction) & $\begin{array}{c}\text { Average relative molecular } \\
\text { mass }\end{array}$ \\
\hline RP-3 real fuel & As shown in Table 1 & 145.5 \\
\hline $\begin{array}{l}\text { four-species surrogate model } \\
\qquad[24]\end{array}$ & $\begin{array}{c}19.1 \% \text { n-decane, } 36.5 \% \\
\text { n-dodecane, } 14.5 \% \\
\text { methyl-cyclohexane, } 29.9 \% \\
\text { n-butylbenzene }\end{array}$ & 143.7 \\
\hline $\begin{array}{l}\text { ten-species surrogate model } \\
\qquad[25]\end{array}$ & $\begin{array}{c}\text { 6\% n-octane, } 10 \% \text { n-decane, } \\
20 \% \text { n-dodecane, } 8 \% \\
\text { n-tridecane } 10 \% \text { n-tetradecane, } \\
10 \% \text { n-Hexadecane, } 20 \% \\
\text { methyl-cyclohexane, } 8 \% \\
\text { 1,3-trans-dimethylcyclopentane, } \\
\text { 5\% propylbenzene, } 3 \% \\
\text { 1-methylnaphthalen }\end{array}$ & 150.1 \\
\hline
\end{tabular}

In the current work, an in-house code programmed with FORTRAN has been developed to compute the thermophysical properties. The core algorithm is the extended corresponding state principle (CSP), it is a widespread change rule in the appropriately non-dimensional properties between different substances [23], as shown in Eq. (1).

$$
f\left(P_{r}, T_{r}, \rho_{r}\right)=0
$$

where

$$
\begin{aligned}
& P_{r}=P / P_{\mathrm{c}} \\
& T_{r}=T / T_{\mathrm{c}} \\
& \rho_{r}=\rho / \rho_{\mathrm{c}}
\end{aligned}
$$

where $P_{c}, T_{c}$ and $\rho_{c}$ are the critical pressure, critical temperature and critical density of the substance, respectively.

Due to its complicated molecular structures leading to the strong polarity and polymeric property, multi-parameter CSP is considered in present study and the effect of the acentric factor and compressibility factor on the thermophysical properties has been included in the link function. The simple compound, propane, which has abundant thermophysical properties data, is selected as the reference substance. 32 parameters MBWR (Modified Benedict-Webb-Rubin) state equation [26] is adopted to describe the P-V-T (pressure, volume, temperature) relation of propane, as shown below:

$$
p_{0}=\sum_{n=1}^{9} a_{n}\left(T_{0}\right) \rho_{0}^{n}+\sum_{n=10}^{15} a_{n}\left(T_{0}\right) \rho_{0}^{2 n-17} e^{-\gamma \rho_{0}^{2}}
$$

Following Eq. (3), the density of surrogate model can be obtained by the link function based on the multi-parameter CSP. TRAPP (Transport properties prediction) method [27] is used for 
calculating the viscosity of surrogate model. It relates the residual viscosity of surrogate model and that of reference substance:

$$
\mu-\mu^{l}=F_{\eta}\left[\mu_{0}-\mu_{0}^{l}\right]+\Delta \mu^{E N S K O G}
$$

where $F_{\eta}$ is the analogical relationship between the residual viscosity of surrogate model and that of reference substance. $\triangle \mu^{E N S K O G}$ is the correction factor due to the hard sphere model hypothesis. The low-pressure viscosity of surrogate model can be computed by Lucas method [28]:

$$
\mu^{l}=\left[0.807 T_{r}^{0.618}-0.357 \exp \left(-0.449 T_{r}\right)+0.34 \exp \left(-4.058 T_{r}\right)+0.018\right] F_{P}^{0} F_{Q}^{0} / \zeta
$$

where

$$
\zeta=0.176\left(\frac{T_{c}}{M^{3} P_{c}^{4}}\right)^{1 / 6}
$$

Similarly, the thermal conductivity can be achieved by TRAPP method, as shown below:

$$
\begin{gathered}
\lambda-\lambda^{l}=F_{\lambda}\left(\lambda_{0}-\lambda_{0}^{l}\right) \\
\lambda^{l}=\left(1.32 C_{p}^{i}+3.741\right) \frac{\mu^{l}}{M}
\end{gathered}
$$

where $F_{\lambda}$ is the analogical relationship between the residual thermal conductivity of surrogate model and that of reference substance, $C_{p}^{i}$ is the specific heat at constant pressure of ideal gas.

According to the thermodynamic principle, the specific heat at constant pressure can be associated with the specific heat at constant volume:

$$
C_{p}=C_{v}+\frac{T}{\rho^{2}}\left(\frac{\partial P}{\partial T}\right)_{\rho}^{2} /\left(\frac{\partial P}{\partial \rho}\right)_{T}
$$

The specific heat at constant volume is obtained by the deviation method, in which the relation of the real property and the corresponding property at low pressure is established.

$$
C_{v}=C_{v}^{l}+\Phi_{C v}(T, \rho)
$$

where $\Phi_{C v}(T, \rho)$ is the deviation function, computed as below:

$$
\Phi_{C v}(T, \rho)=T \int_{\infty}^{V}\left(\frac{\partial^{2} P}{\partial T^{2}}\right)_{V} d V
$$

Variation of the computed density, viscosity, thermal conductivity and specific heat with temperature at different pressure of the four-species surrogate model (4CM) and ten-species surrogate model (10CM) are shown in Figs.1-4, together with those obtained by experiments [29-31]. 

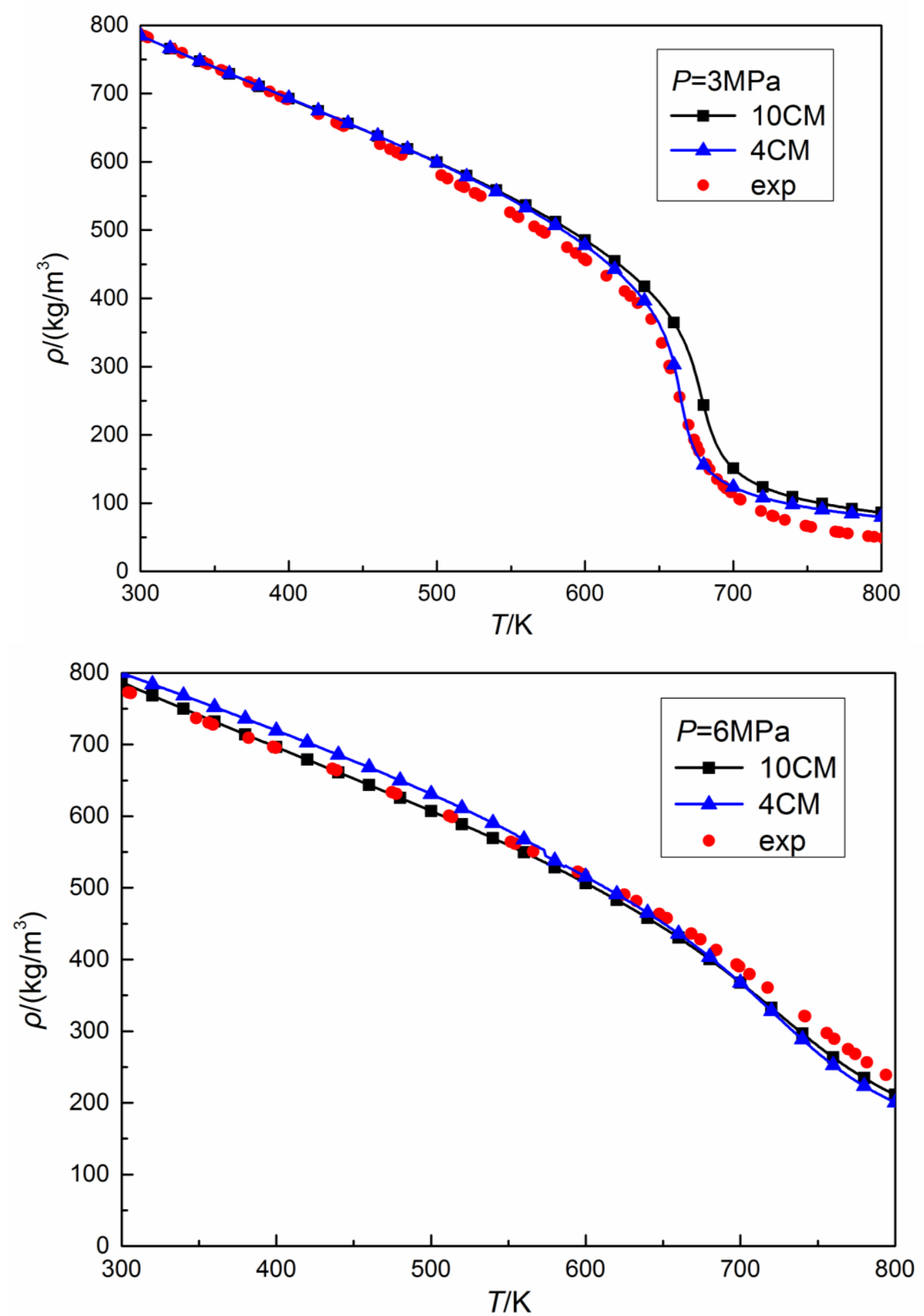

Fig. 1. Comparison of calculation data and experiment data of density at constant pressure for different surrogate models. 

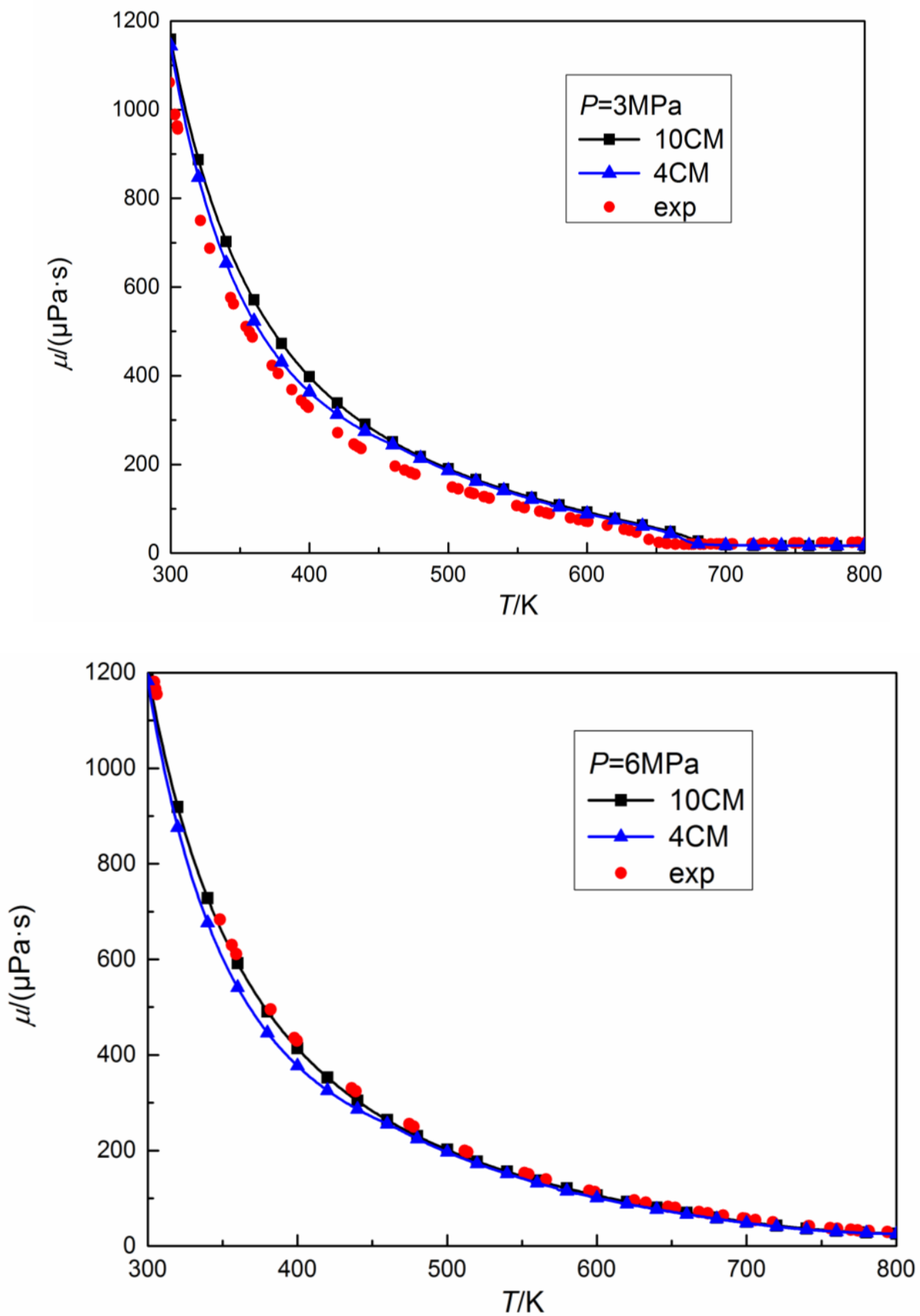

Fig. 2. Comparison of calculation data and experiment data of viscosity at constant pressure for different surrogate models. 

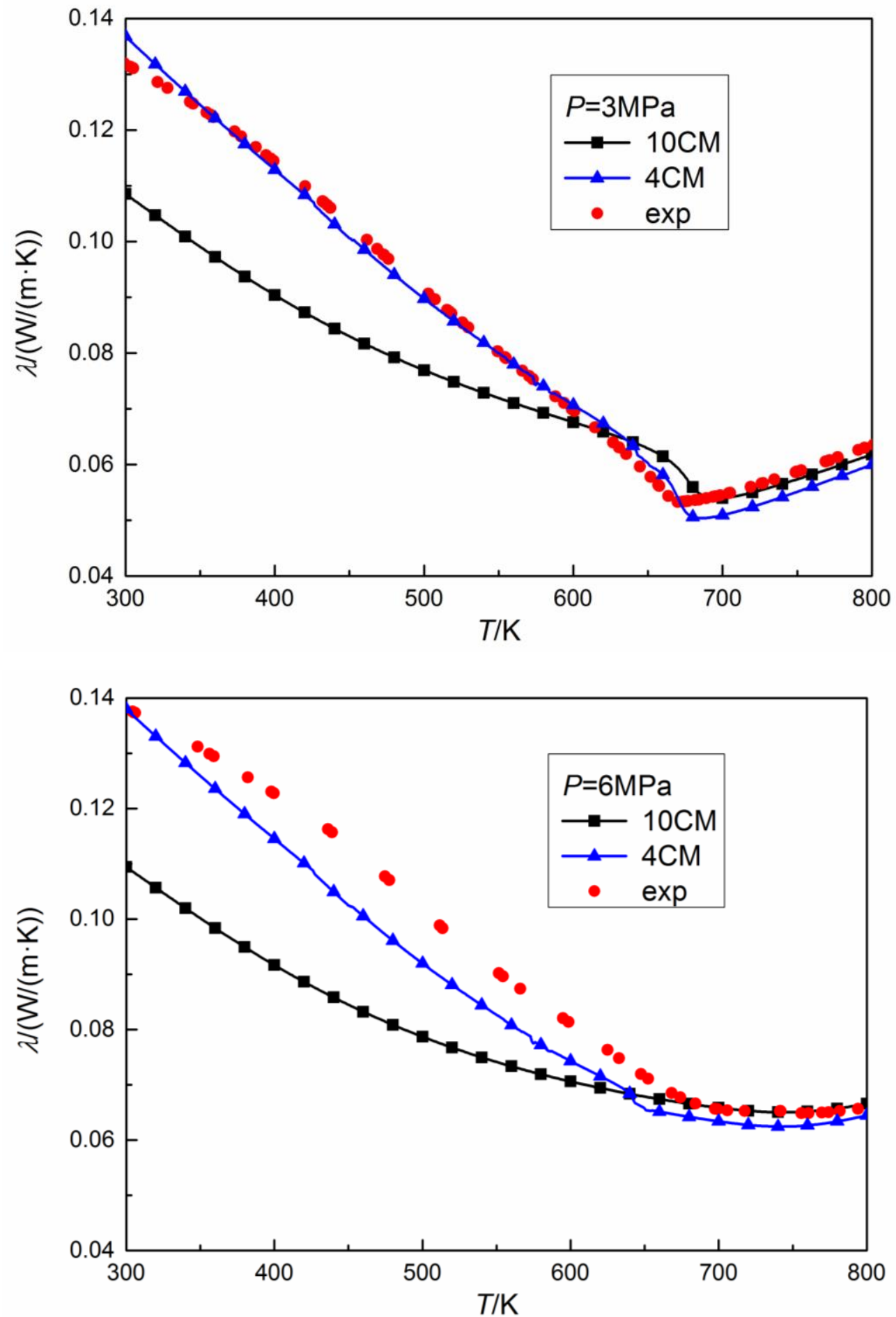

Fig. 3. Comparison of calculation data and experiment data of thermal conductivity at constant pressure for different surrogate models. 

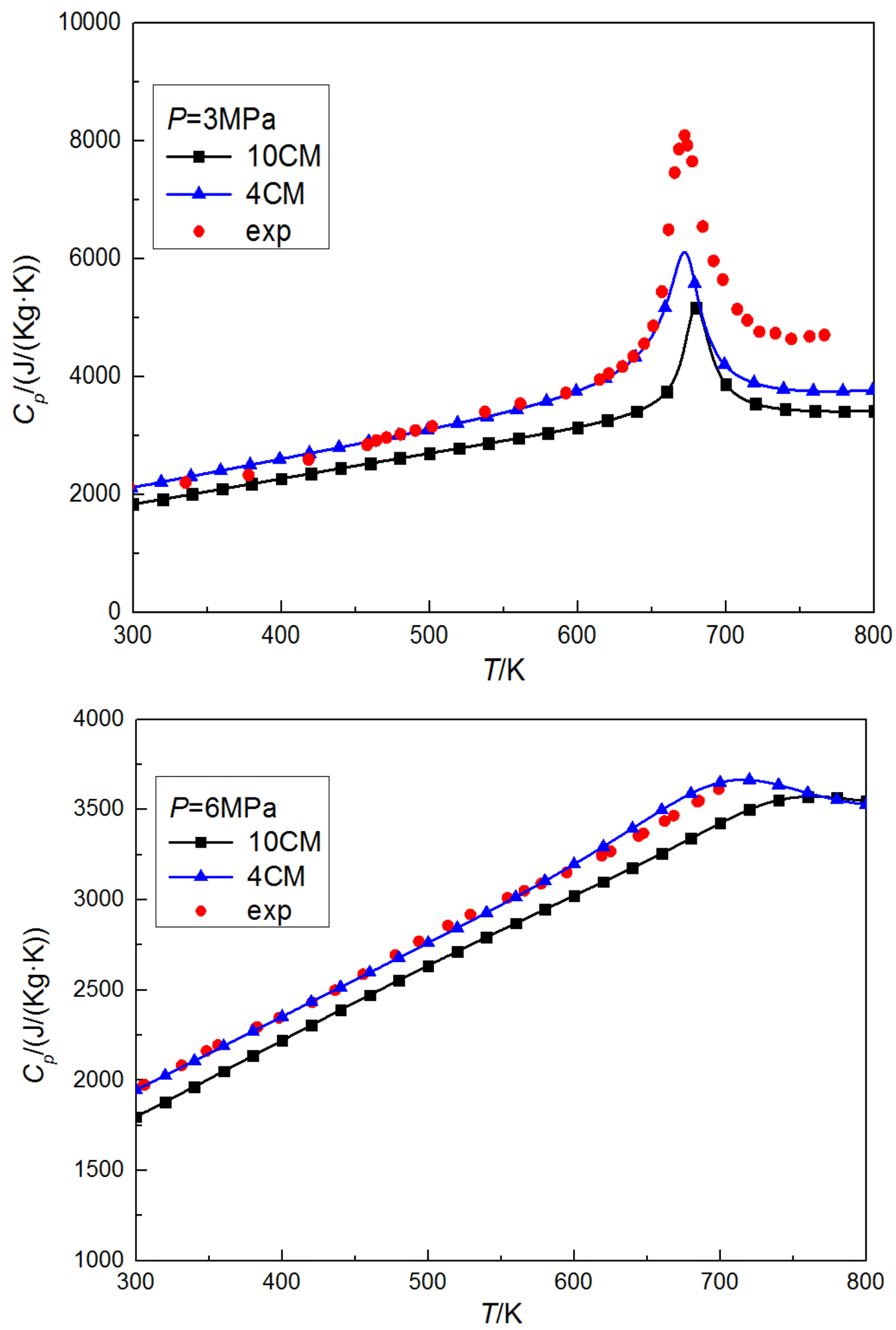

Fig. 4. Comparison of calculation data and experiment data of specific heat at constant pressure for different surrogate models.

It can be seen clearly from Figs. 1-4, the four-species surrogate model would be the best candidate in predicting the thermophysical properties, especially for the thermal conductivity and specific heat. Considering that the pyrolysis reaction at the temperature higher than $700 \mathrm{~K}$ which may lead to the larger inaccuracy of the measured values, the experimental results in the range 
from $300 \mathrm{~K}$ to $700 \mathrm{~K}$ are selected for error analysis. The relative deviation of four thermophysical parameters by four-species surrogate model at different pressures is shown in Table 3. The RMS value means the root-mean-square value at all of the temperature points used in the error analysis here and the relative deviation is the ratio of the absolute deviation and the experimental value.

Table 3 Relative deviations of four-species surrogate model at different pressures.

\begin{tabular}{cccccc}
\hline & \multicolumn{2}{c}{$3 \mathrm{MPa}$} & \multicolumn{2}{c}{$6 \mathrm{MPa}$} & \multirow{2}{*}{ Average RMS } \\
& RMS & Max. & RMS & Max. & \\
\hline Density & 0.031 & 0.084 & 0.03 & 0.054 & 0.03 \\
Viscosity & 0.109 & 0.18 & 0.11 & 0.15 & 0.11 \\
Conductivity & 0.02 & 0.07 & 0.06 & 0.09 & 0.04 \\
Specific heat & 0.1 & 0.15 & 0.01 & 0.03 & 0.06 \\
\hline
\end{tabular}

The average RMS relative deviations of the density, viscosity, conductivity and specific heat obtained by four-species surrogate model are $0.03,0.11,0.04$ and 0.06 , respectively. It has been verified that the slight pyrolysis reaction of RP-3 starts to occur when the temperature is larger than $673 \mathrm{~K}$ and its reaction rate increases exponentially with the increase of temperature [2]. The pyrolysis reaction makes one large molecule cracking into several small molecules, accompanied by the obvious chemical heat absorption and hence leads to the reduced measured density, the reduced measured viscosity and the larger measured specific heat in the vicinity of pseudo-critical temperature, limited to the measurement method [29]. The computed critical temperature and pressure of four-species surrogate model are $643.5 \mathrm{~K}$ and $2.43 \mathrm{MPa}$, respectively, while the corresponding measured values of RP-3 are $645.04 \mathrm{~K}$ and $2.33 \mathrm{MPa}$ [32], respectively. By comprehensive considering of the effect of chemical factor on experimental results, it is indicated that the four-species surrogate model with proposed thermophysical properties computation code is reliable.

\section{Computational methodology}

\subsection{Test case description}

Fig. 5 shows the sample profile of computation model. A long round tube is used in the current study. In order to eliminate the entrance and exit effect as well as to get the fully developed turbulence flow, the adiabatic sections are set up before and after the uniformly-heated section in the middle of the whole tube. As diameter increases, the length of round tube is adjusted properly to satisfy the proportional requirement. Table 4 summarizes the detailed information of five kinds of computation models used in the current work. 


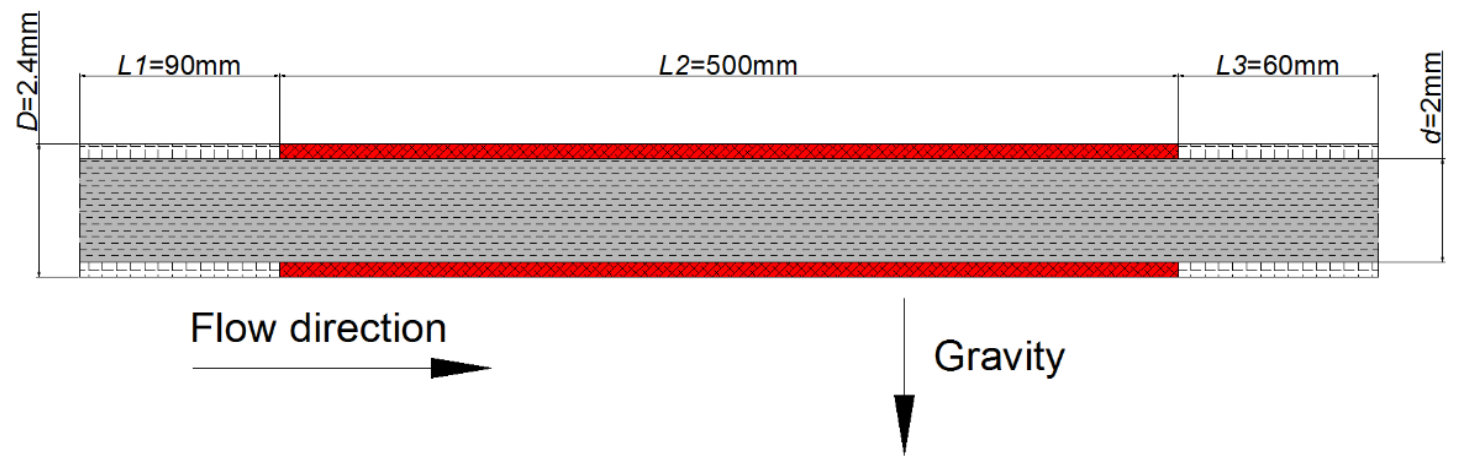

Fig. 5. Schematic flow diagram of the computation model.

Table 4 Detailed information of computation models.

\begin{tabular}{ccccccc}
\hline No. & $\begin{array}{c}\text { Outer } \\
\text { diameter } \\
D / \mathrm{mm}\end{array}$ & $\begin{array}{c}\text { Inner } \\
\text { diameter } \\
d / \mathrm{mm}\end{array}$ & $\begin{array}{c}\text { Inlet adiabatic } \\
\text { section length } \\
L 1 / \mathrm{mm}\end{array}$ & $\begin{array}{c}\text { Heating } \\
\text { section } \\
\text { length } \\
\text { L2/mm }\end{array}$ & $\begin{array}{c}\text { Outlet adiabatic } \\
\text { section length } \\
L 3 / \mathrm{mm}\end{array}$ & $\begin{array}{c}\text { Total } \\
\text { length } \\
L / \mathrm{mm}\end{array}$ \\
\hline 1 & 2.4 & 2 & 90 & 500 & 60 & 650 \\
2 & 4.4 & 4 & 180 & 1000 & 120 & 1300 \\
3 & 6.4 & 6 & 270 & 1500 & 180 & 1950 \\
4 & 8.4 & 8 & 360 & 2000 & 240 & 2600 \\
5 & 10.4 & 10 & 450 & 2500 & 300 & 3250 \\
\hline
\end{tabular}

Due to the tiny effect of thermal conduction in tube wall on supercritical heat transfer of hydrocarbon fuel [33], the wall thickness in different models is fixed at $0.2 \mathrm{~mm}$ and the structural strength of tube wall is not considered. In the current study, conjugate heat transfer simulation is carried out in order to compare with the actual experimental cases. 321 stainless steel is selected as the tube material, whose thermal conductivity changes as the function of temperature is shown below:

$$
\lambda=15.03543+0.01178 \times(T-273.15)+4.20854 \times 10^{-6} \times(T-273.15)^{2}
$$

\subsection{Governing equations}

The tensor forms of the steady governing equations, including mass conservation, momentum conservation and energy conservation are shown as follows:

Continuity equation:

$$
\frac{\partial\left(\rho \mathrm{u}_{i}\right)}{\partial x_{i}}=0
$$

Momentum equation:

$$
\frac{\partial}{\partial x_{j}}\left(\rho \mathrm{u}_{i} \mathrm{u}_{j}\right)=\rho \mathrm{g}_{i}-\frac{\partial P}{\partial x_{i}}+\frac{\partial \tau_{i j}}{\partial x_{j}}+\frac{\partial}{\partial x_{j}}\left(-\rho \overline{u_{i}^{\prime} u_{j}^{\prime}}\right)
$$

\section{Energy equation:}




$$
\frac{\partial}{\partial x_{i}}\left(\rho u_{i} H\right)=\frac{\partial}{\partial x_{i}}\left(\lambda \frac{\partial T}{\partial x_{i}}+\frac{\mu_{t}}{\operatorname{Pr}_{t}} \frac{\partial H}{\partial x_{i}}\right)
$$

where $P r_{t}$ is turbulent Prandtl number taken as 0.85 and $H$ is enthalpy. It is important to note that the temperature of thermal pyrolysis onset is larger than that of heat transfer deterioration onset and the effect of thermal pyrolysis can be ignored.

The following formula is used to calculate the Nusselt number:

$$
N u=h d / \lambda
$$

where the convective heat transfer coefficient is calculated as below:

$$
h=q /\left(T_{w}-T_{b}\right)
$$

The bulk temperature is obtained as below:

$$
T_{b}=\frac{\int_{A} \rho u C_{p} T d A}{\int_{A} \rho u C_{p} d A}
$$

Turbulence modelling is significantly influenced by severe change of the physical property under supercritical pressure. It was widely acknowledged that the low-Reynolds number $k-\varepsilon$ type turbulence models have the feature to predict well the variable properties effect and buoyancy effect at supercritical pressure [35]. Recently, the effectiveness of Spalart-Allmaras turbulence model in predicting flow and heat transfer of supercritical methane has been validated [34]. The comparison study of several turbulence models in predict supercritical heat transfer has been carried out [35,36] and LS model (proposed by Launder and Sharma) [37] has been found to perform well in predicting supercritical heat transfer, therefore LS low-Reynolds number eddy viscosity turbulence model is selected in the present study.

The constitutive equation and the turbulence transport equation are shown as below:

\section{Constitutive equation:}

$$
\mu_{t}=\rho C_{\mu} f_{\mu} \frac{k^{2}}{\varepsilon}
$$

Turbulent kinetic energy:

$$
\frac{\partial}{\partial x_{i}}\left(\rho \mathrm{u}_{i} \mathrm{k}\right)=\frac{\partial}{\partial x_{i}}\left[\left(\mu+\frac{\mu_{t}}{\sigma_{k}}\right) \frac{\partial k}{\partial x_{i}}\right]-\rho \overline{u_{i}^{\prime} u_{j}^{\prime}} \frac{\partial u_{i}}{\partial x_{j}}+G_{k}-\rho \varepsilon-S_{k}
$$

The rate of the turbulent dissipation:

$$
\frac{\partial}{\partial x_{i}}\left(\rho u_{i} \varepsilon\right)=\frac{\partial}{\partial x_{i}}\left[\left(\mu+\frac{\mu_{t}}{\sigma_{\varepsilon}}\right) \frac{\partial \varepsilon}{\partial x_{i}}\right]+C_{\varepsilon 1} f_{1} \frac{\varepsilon}{k}\left(-\rho \overline{u_{i}^{\prime} u_{j}^{\prime}} \frac{\partial u_{i}}{\partial x_{j}}+g_{i} \overline{\rho^{\prime} u_{i}^{\prime}}\right)-C_{\varepsilon 2} f_{2} \rho \frac{\varepsilon^{2}}{k}+S_{\varepsilon}
$$

\section{Model constants:}

$$
C_{\mu}=0.09, C_{\varepsilon 1}=1.44, C_{\varepsilon 2}=1.92, \sigma_{k}=1.0, \sigma_{\varepsilon}=1.3
$$

The summary of the functions, additional terms and boundary conditions is listed in Table 5 .

Table 5 Summary of the functions, additional terms and boundary conditions on the wall in the LS model. [37]

\begin{tabular}{llllll}
\hline$f_{1}$ & $f_{2}$ & $f_{\mu}$ & $S_{k}$ & $S_{\varepsilon}$ & Boundary condition \\
\hline
\end{tabular}


$1.01-0.3 \exp \left(-\mathrm{Re}_{t}^{2}\right) \quad \exp \left[\frac{-3.4}{\left(1+\mathrm{Re}_{t} / 50\right)^{2}}\right] 2 u\left(\frac{\partial \sqrt{k}}{\partial y}\right)^{2} 2 m \frac{m_{t}}{r}\left(\frac{\partial^{2} V}{\partial x^{2}}\right)^{2}\left(\frac{\partial k}{\partial y}\right)_{w}=0, e_{w}=0$

\subsection{Computational mesh}

Three dimensional structural mesh is built by ANSYS ICEM using the top-down structural block division method to discretize all of the computational domain covering the adiabatic and heated sections along the length direction, ranging from the fluid domain to the tube wall. The special attention is given to the fined mesh near the wall to catch the rapid change of fluid properties in the boundary layer. The grid independent solution is inspected by trials and comparisons in each run. As continuously refining the mesh at different directions, the temperature and velocity at monitored locations are observed, as shown in Fig. 6. The calculation conditions in Fig. 6 are: diameter is $4 \mathrm{~mm}$, pressure is $3 \mathrm{MPa}$, heat flux is $500 \mathrm{~kW} / \mathrm{m}^{2}$ and mass flux is $704 \mathrm{~kg} /\left(\mathrm{m}^{2} \cdot \mathrm{s}\right)$. The monitored location is the center point of outlet surface. It can be seen that the change of monitored quantities with the number of cells fades and as the grid number reaches to 757492 , the change rate is less than $1 \%$, implying that the grid independent solution is achieved. The numbers of the optimal mesh of grids in models 1-5, typically, are 757492, 1763637, 2334950, 3529687 and 4412712, respectively. The distance between two adjacent nodes grows by a factor of 1.05 as staying away from the wall in the fluid domain. Moreover, the first node close to the wall has $y^{+}<1$ in all cases, in order to compute from the viscous sub-layer.

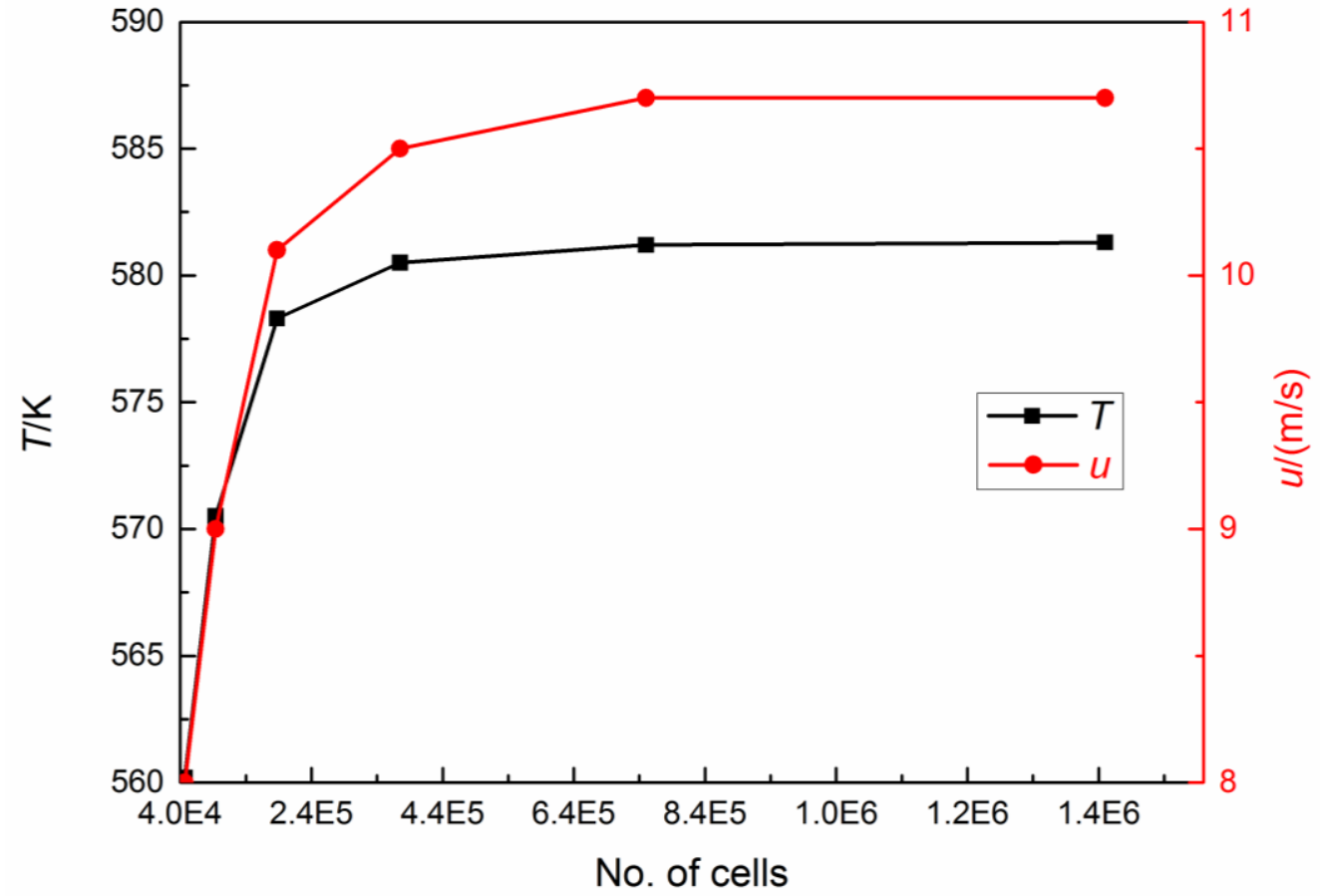

Fig. 6. Variations of temperature and velocity with mesh numbers.

Fig. 7 gives the mesh map in the cross section of tube. In the current study, the bottom generatrix of tube (point 0 ) is regarded as 0 degree circumference angle and the top generatrix (point 6) is 180 degree angle. The five points in the cross section, corresponding to five 
generatrices, are uniformly located in the half circumference, as shown in Fig. 7.

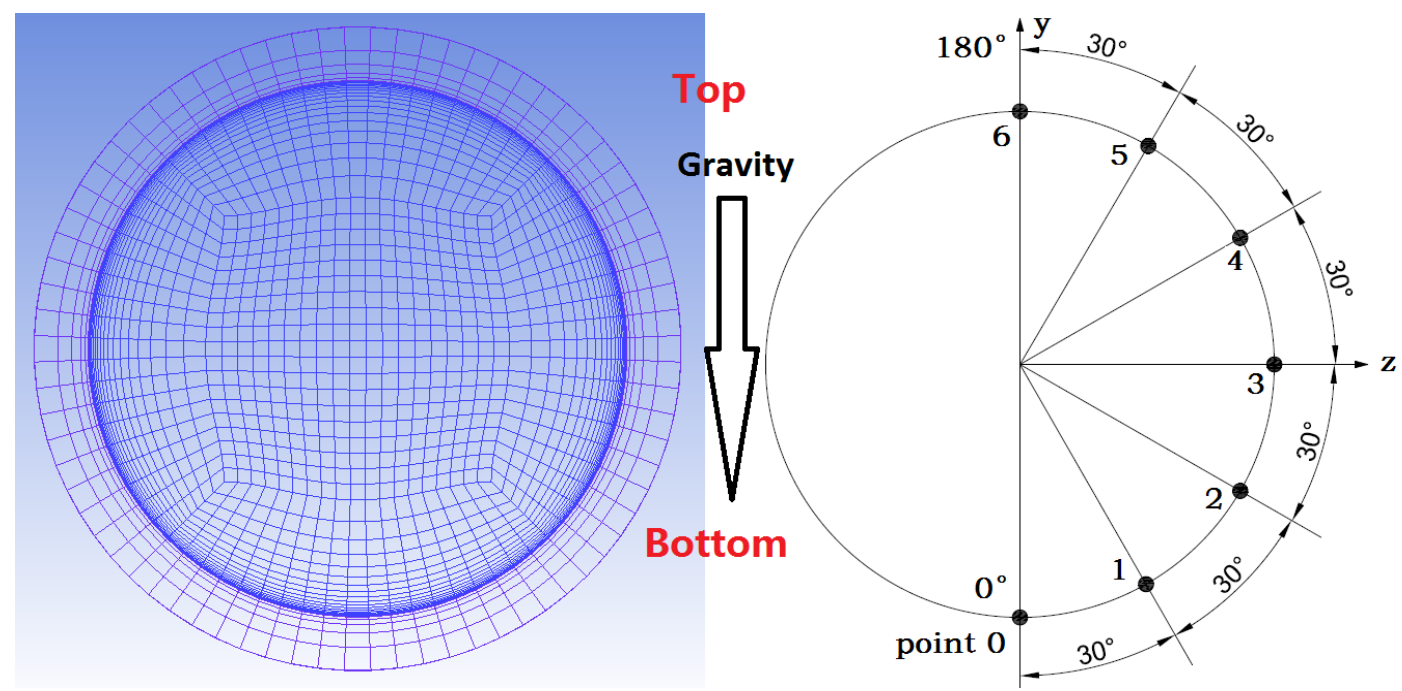

Fig. 7. Mesh map in the cross section of tube.

\subsection{Numerical method}

The pressure-based segregated solver with SIMPLEC (Semi-Implicit Method for Pressure Linked Equations-Consistent) scheme in ANSYS Fluent [38] is used as the pressure-velocity coupling method to accelerate the convergence. The Least Squares Cell-Based Gradient Evaluation is applied for modelling the gradient of the convection and diffusion terms in the conservation equations and the STANDARD scheme is adopted to interpolate the pressure values at the face of grid cell. The second order upwind scheme is used for computing the face values in the momentum and energy equations to improve the accuracy of calculation, and the first order upwind scheme is used for turbulence equation to enhance the robustness. The absolute convergence criterion of residual for all of the equations is set to be less than $10^{-6}$.

The mass-flow inlet type is set for inlet boundary condition in which the inlet mass flux and total temperature are specified and the pressure at the outlet of pipe is given for outlet boundary condition. The constant heat flux is imposed on the outer wall of uniformly-heated section and is served as wall boundary condition. It is noted that the value of the wall heat flux which appears in the paper is the heat flux at inner wall for the comparison of experimental heat flux, due to that the experiment gave the heat flux at inner wall.

\subsection{Model validation}

Prior to conducting the aimed computations, it is necessary to validate the computational model and the developed code. In the current work, since there is no suitable experimental results of hydrocarbon fuel in the horizontal tube, normal heat transfer data of supercritical RP-3 flowing in a vertically heated $1.8 \mathrm{~mm}$ ID (short for the inner diameter) tube [2] is selected. In order to compute efficiently, a 2D axisymmetric mesh is made in the vertical flow and the detailed information about mesh can be seen in the reference [36]. Considering that there is no suitable heat transfer deterioration experimental results of RP-3, the experiment about heat transfer deterioration of supercritical n-decane in a uniformly heated $2 \mathrm{~mm}$ ID tube [39] is simulated. Table 6 shows the list of validation conditions in the experiments. 
Table 6 List of validation conditions in the experiments

\begin{tabular}{|c|c|c|c|c|c|c|}
\hline No. & Pressure/MPa & $\begin{array}{c}\text { Inlet } \\
\text { temperature/ } \\
\mathrm{K} \\
\end{array}$ & $\begin{array}{c}\text { Mass } \\
\text { rate/(g/s) }\end{array}$ & $\begin{array}{c}\text { Heat } \\
\text { flux } /\left(\mathrm{kW} / \mathrm{m}^{2}\right)\end{array}$ & $\mathrm{ID} / \mathrm{mm}$ & $\begin{array}{c}\text { Heat transfer } \\
\text { type }\end{array}$ \\
\hline Run 1 & 5 & 373 & 3 & 300 & 1.8 & $\begin{array}{c}\text { Normal heat } \\
\text { transfer }\end{array}$ \\
\hline Run 2 & 5 & 373 & 3 & 400 & 1.8 & $\begin{array}{l}\text { Normal heat } \\
\text { transfer }\end{array}$ \\
\hline Run 3 & 3 & 423 & 3 & 218 & 1.8 & $\begin{array}{l}\text { Heat transfer } \\
\text { deterioration }\end{array}$ \\
\hline Run 4 & 3 & 423 & 3 & 279 & 2 & $\begin{array}{l}\text { Heat transfer } \\
\text { deterioration }\end{array}$ \\
\hline Run 5 & 3 & 423 & 3 & 306 & 2 & $\begin{array}{l}\text { Heat transfer } \\
\text { deterioration }\end{array}$ \\
\hline
\end{tabular}

The calculated wall temperatures of supercritical heat transfer including both normal heat transfer and heat transfer deterioration are compared with the corresponding experimental data as shown in Fig. 8. The axial relative distance $x /(L 2)$ is the ratio of the distance from the inlet of the heating section to the length of the heated section.

It can be seen from Fig. 8, the simulated results can qualitatively predict well the experimental data in both normal heat transfer and heat transfer deterioration modes. In normal heat transfer mode, very small difference between the calculated result and experimental data is mainly focused on local heat transfer deterioration at the beginning of the heated section. According to the reference [2], the local heat transfer deterioration is caused by the development of the thermal boundary layer and the decreasing thermal conductivity, further leading to the thermal resistance layer with very slow velocity. Hence, the deviations in the thermophysical property model and turbulence model may result in the worse capture of local heat transfer deterioration. In heat transfer deterioration mode, the maximum deviation of $7.48 \%$ occurs in run 5 where the average deviation is less than $3.45 \%$. The distinction between the calculated result and experimental data appears as the wall temperature is larger than $760 \mathrm{~K}$ which may be caused by the endothermic pyrolysis reaction [40]. Overall, it is implied that the numerical method used in current paper is reliable. 


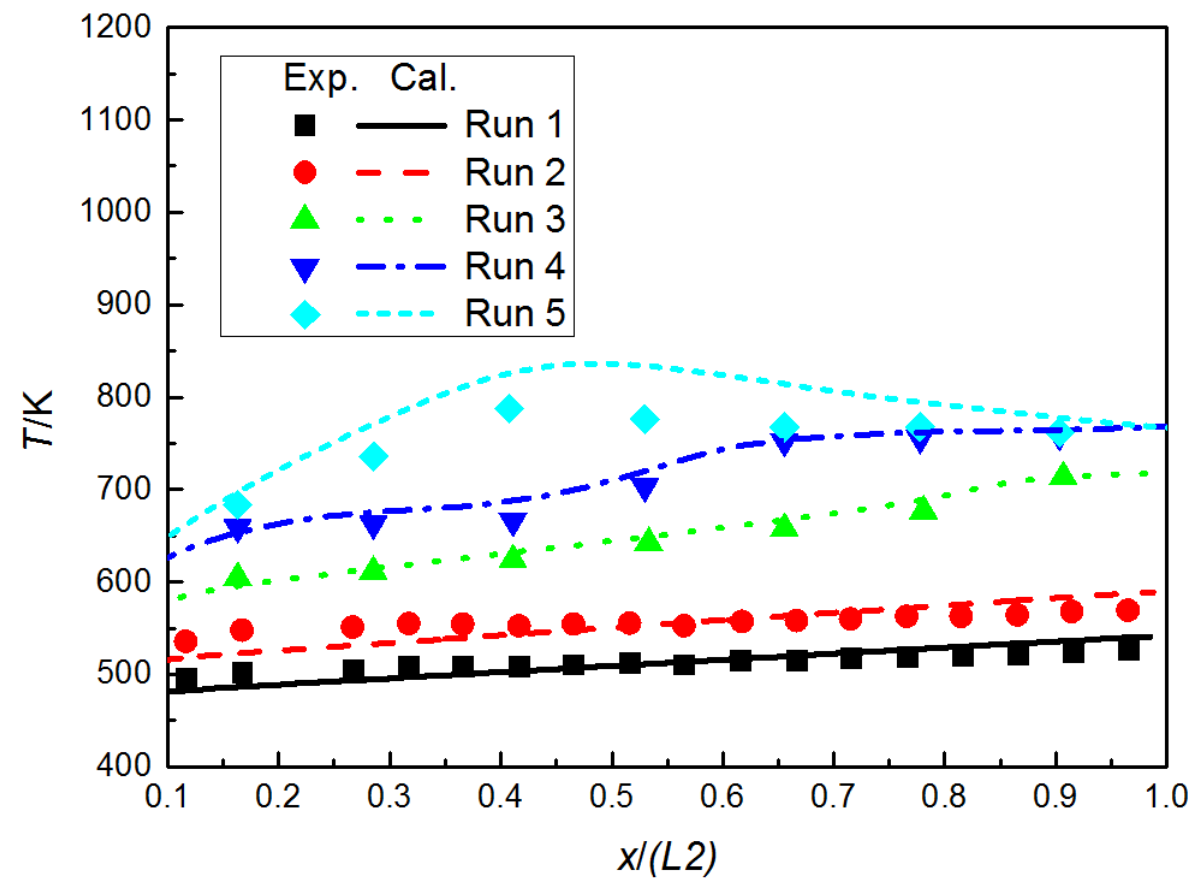

Fig. 8. Comparisons between calculated wall temperature and experimental results

\section{Results and discussion}

\subsection{Heat transfer characteristics in the horizontal tube}

The working pressure of the actual cooling process in both regenerative cooling and CCA covers the broad range at supercritical pressure so $3 \mathrm{MPa}$ is selected as the operating pressure of the current study. When the pressure is closer to critical pressure (2.43 MPa), the effect of variation of properties of RP-3 on heat transfer is more obvious. Although the selection of heat flux and mass flux in the current study may not cover the actual extreme working conditions, the insight of supercritical peculiar phenomenon can be investigated due to that the characteristics of transcritical alteration in the real cooling process, including large heat flux to mass flux ratio, are not lost. Typical calculation conditions are: pressure is $3 \mathrm{MPa}$, heat flux is $500 \mathrm{~kW} / \mathrm{m}^{2}$ and mass flux is $704 \mathrm{~kg} /\left(\mathrm{m}^{2} \cdot \mathrm{s}\right)$. Fig. 9 gives the variations of temperature and heat transfer coefficient with the bulk enthalpy along generatrices 0-6, corresponding to points 0-6 (Fig. 7) in $2 \mathrm{~mm}$ diameter tube. 


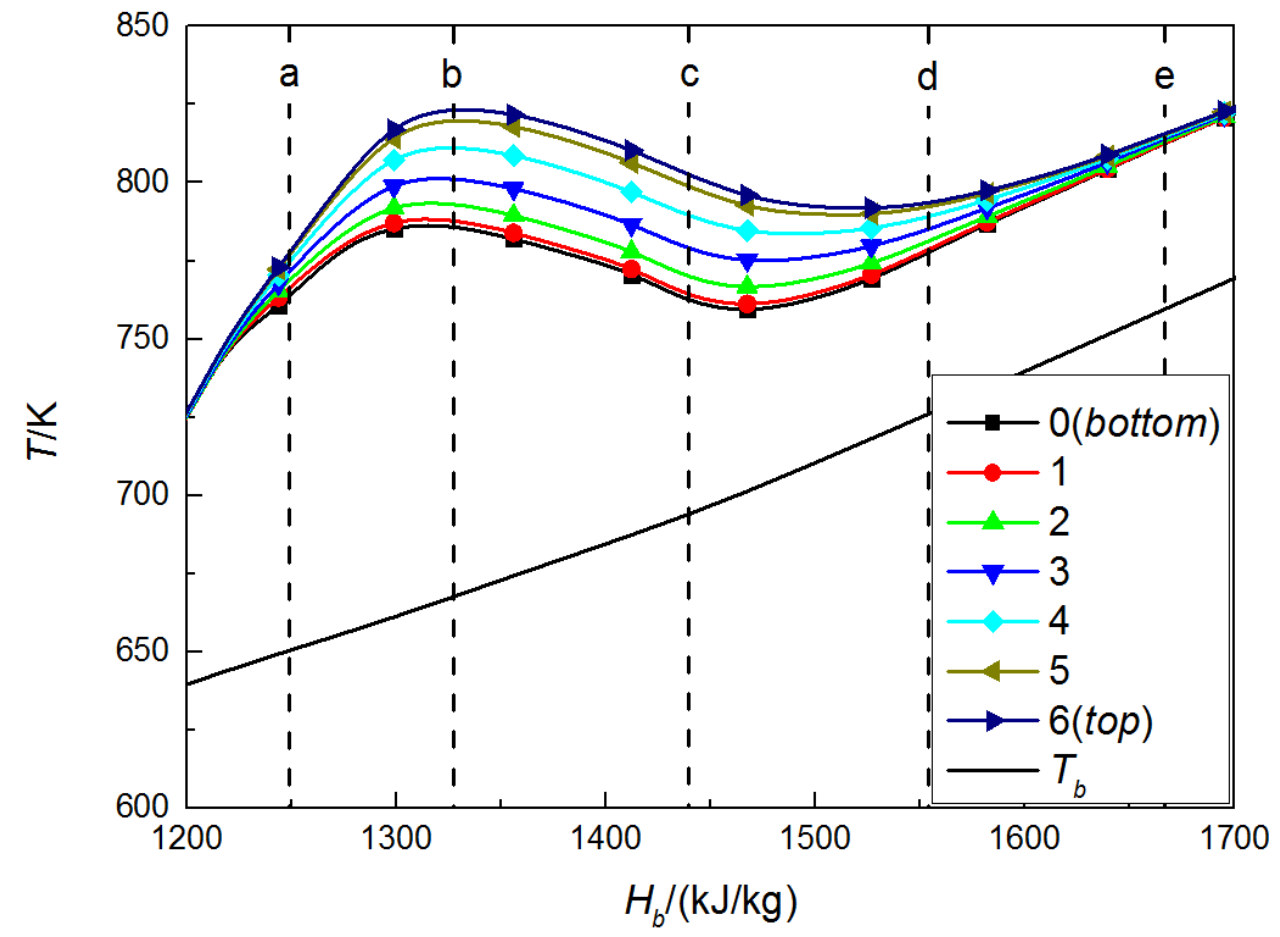

(a) temperature

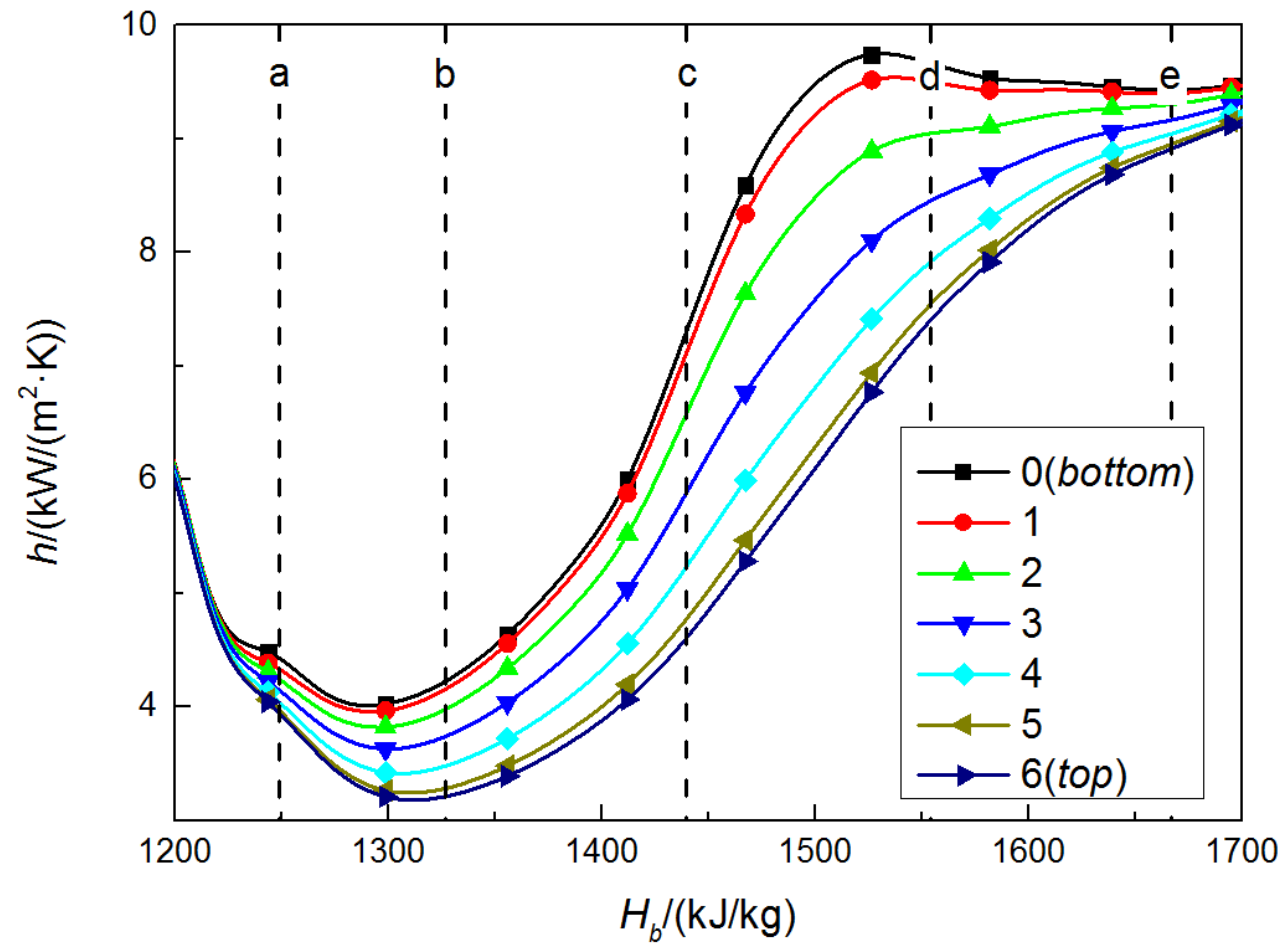

(b) heat transfer coefficient

Fig. 9. Variations of temperature and heat transfer coefficient along generatrices 0-6 in $2 \mathrm{~mm}$ tube.

In Fig. 9, at each generatrix, the temperature initially increases until reaches to a peak, and then decreases for a while followed by the monotonic linear increase. It has been observed that as the pseudo-critical temperature is below the inner wall temperature and above the fluid bulk temperature, heat transfer deterioration occurs caused by very low heat exchange between the flow 
core and the wall. As the fluid bulk temperature starts to exceed the pseudo-critical temperature where the drastic variation of thermophysical properties weaken, heat transfer deterioration is reduced and heat exchange between the flow core and the wall begins to recover. The valley of heat transfer coefficient corresponds to the peak of temperature and then heat transfer coefficient picks up. At a certain cross section, with the increase of circumference angle, temperature increases and heat transfer coefficient decreases, replying a more severe heat transfer deterioration. There exists a large temperature difference up to $50 \mathrm{~K}$ between the top and bottom generatrices, and heat transfer coefficient at the bottom generatrix can reach to 2 times of that at the top generatrix.

The following detailed analysis is used for deep accounting for the above phenomenon. According to the locations of heat transfer deterioration and heat transfer recovery, five cross sections (a-e) are selected, corresponding to five enthalpies, $1244 \mathrm{~kJ} / \mathrm{kg}, 1327 \mathrm{~kJ} / \mathrm{kg}, 1440 \mathrm{~kJ} / \mathrm{kg}$, $1554 \mathrm{~kJ} / \mathrm{kg}$ and $1667 \mathrm{~kJ} / \mathrm{kg}$, respectively. As the bulk enthalpy is $1244 \mathrm{~kJ} / \mathrm{kg}$, heat transfer deterioration starts to appear, and as the bulk enthalpy is $1440 \mathrm{~kJ} / \mathrm{kg}$, heat transfer is impaired most seriously. Heat transfer deterioration is reduced at $1554 \mathrm{~kJ} / \mathrm{kg}$, and heat transfer enhancement and recovery can be seen at $1554 \mathrm{~kJ} / \mathrm{kg}$ and $1667 \mathrm{~kJ} / \mathrm{kg}$. Fig. 10 shows the radial distribution of density, temperature, axial velocity and turbulent kinetic energy along the vertical diameter line $(y$ axis) at the selected five cross sections.

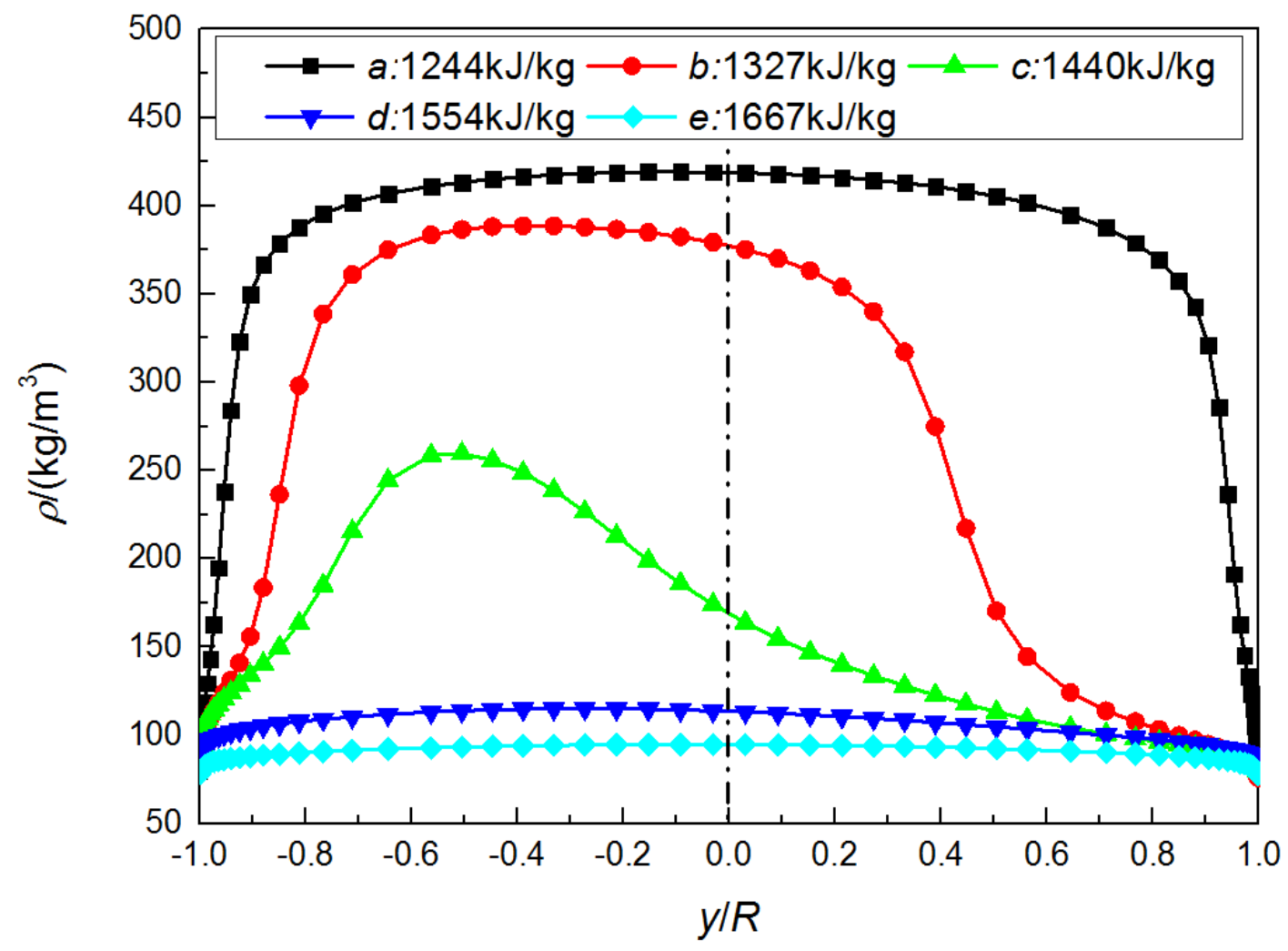

(a) density 


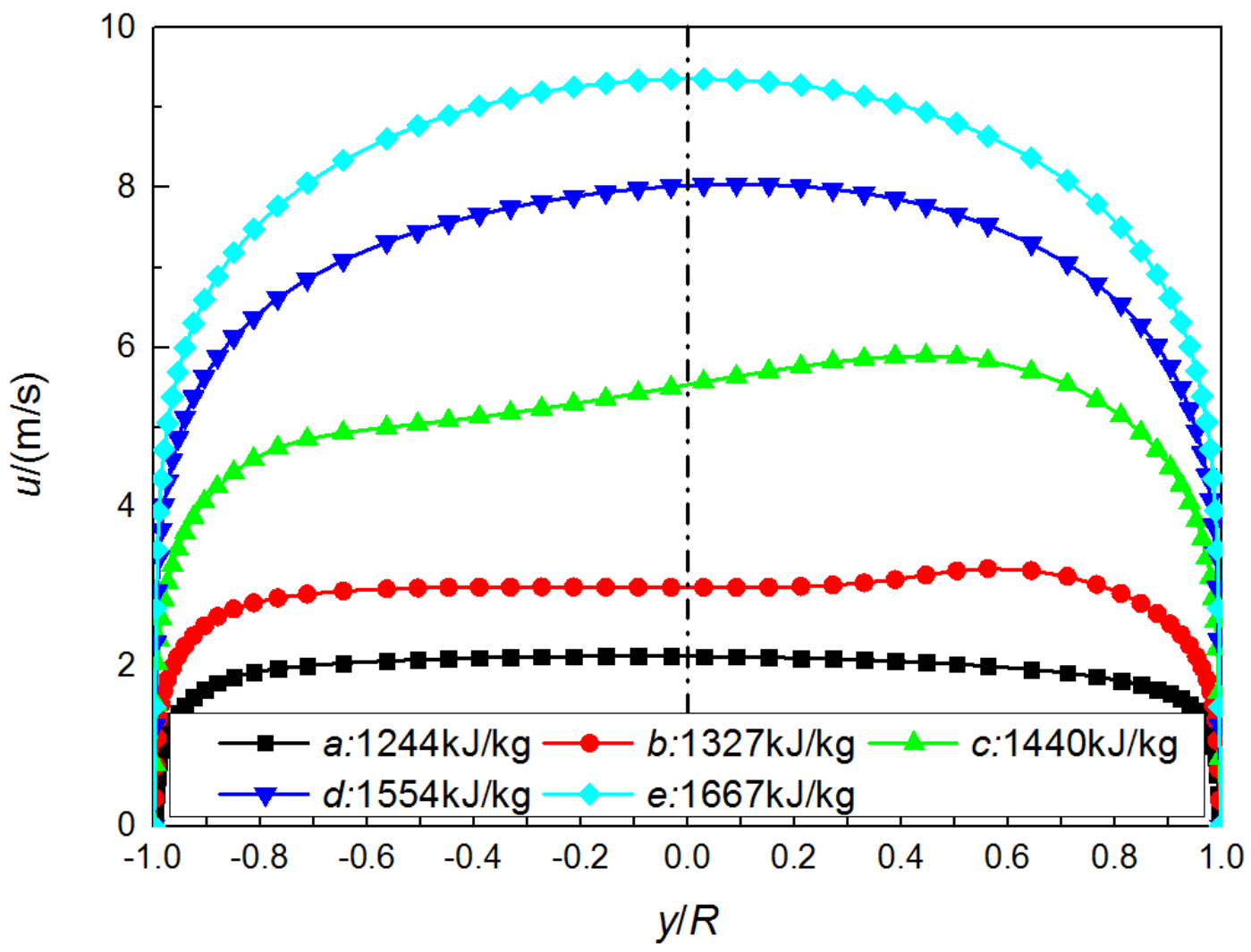

(b) axial velocity

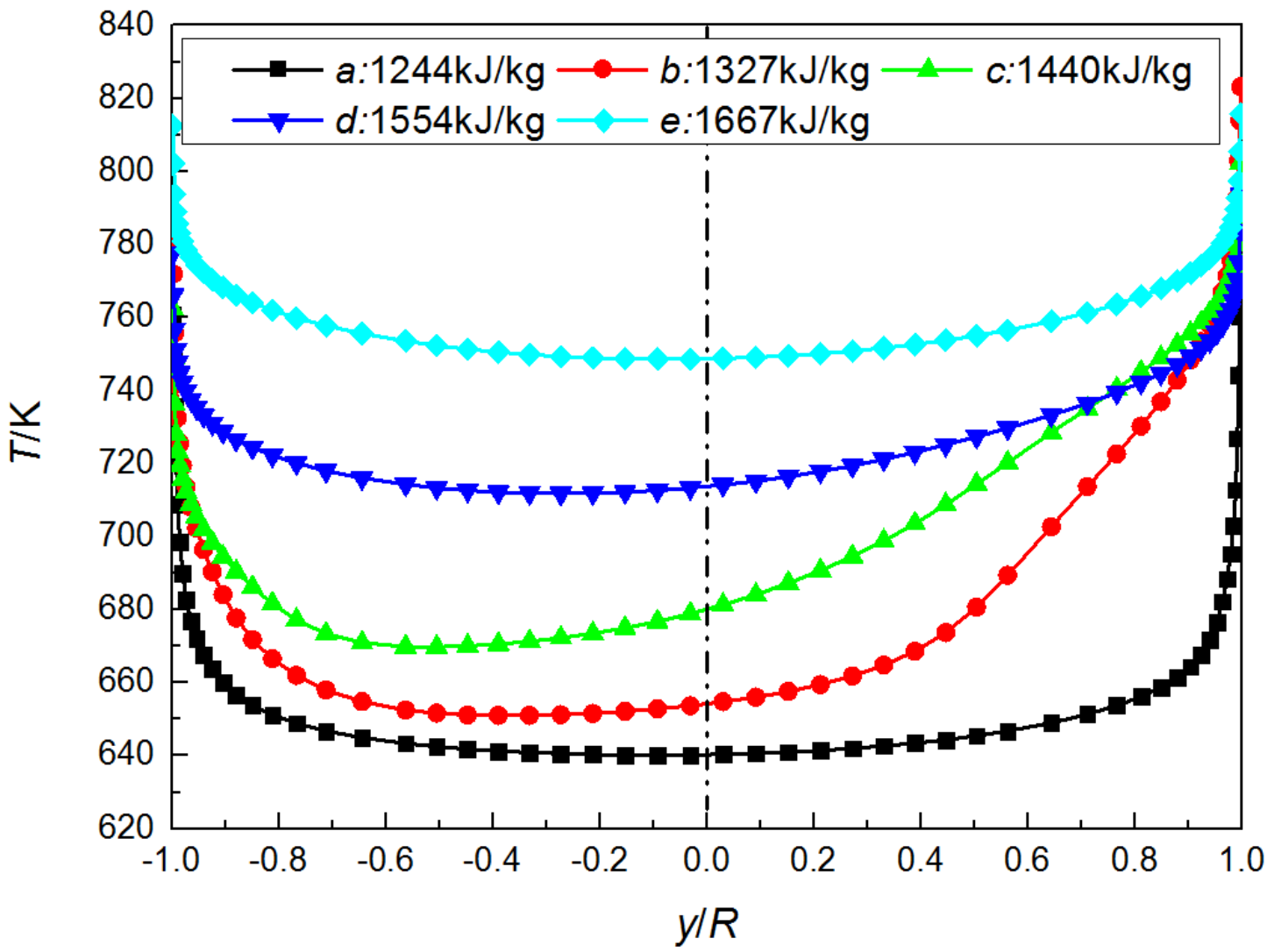

(c) temperature 


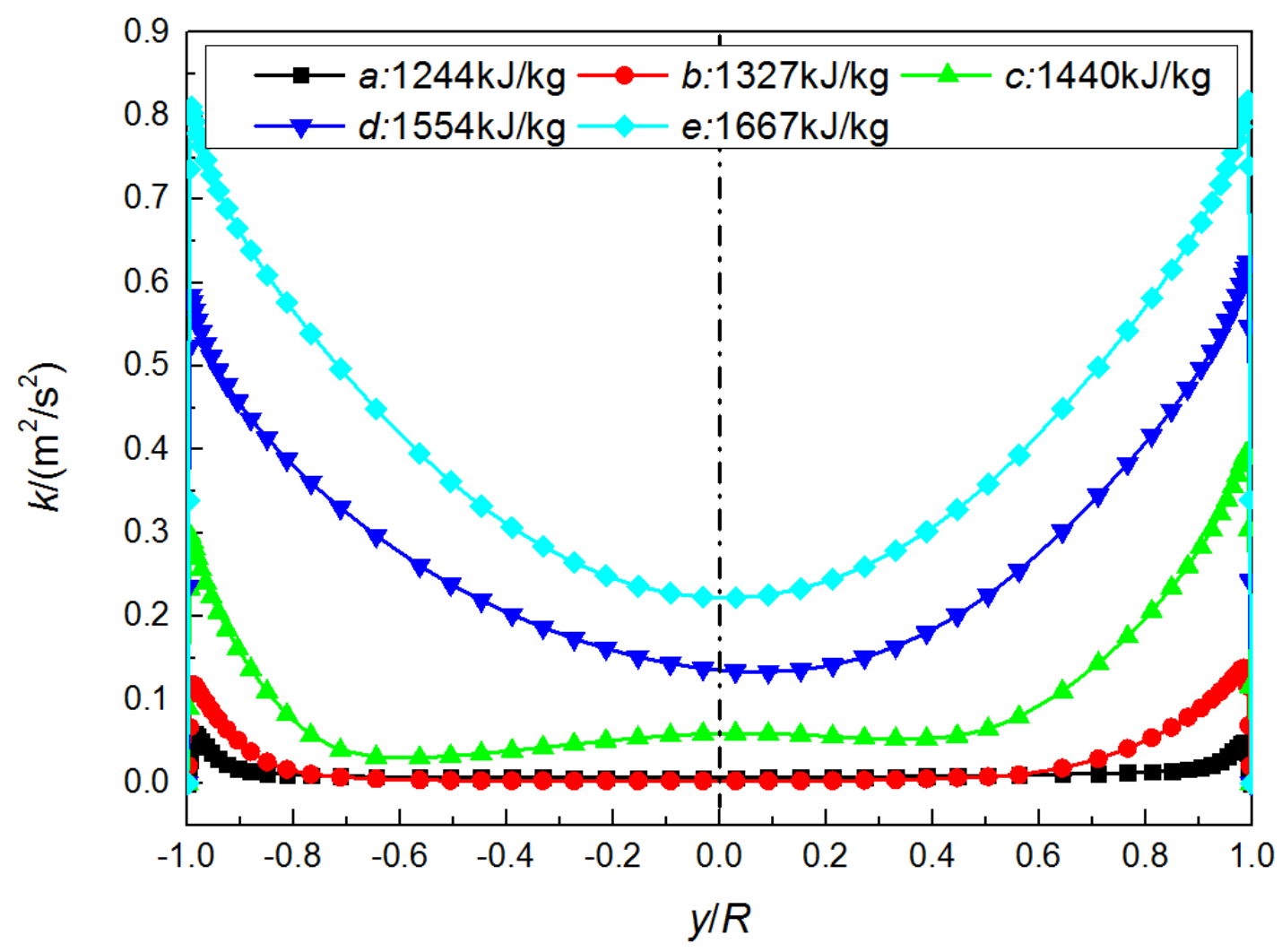

(d) turbulent kinetic energy

Fig. 10. Radial distributions of density, axial velocity, temperature and turbulent kinetic energy along the vertical diameter line.

In Fig. 10(a), due to the heated wall, the density at the core is higher than that near the wall. At section $a$, the distribution of density is almost symmetric with the center line. As flowing downstream, the distribution of density is not symmetrical with the center line, that is, in top half part, a large density variation occurs at the flow core whereas in the bottom half part, it happens away from the flow core, as a result, the buoyancy has more obvious effect at top half than at bottom half, resulting in that the temperature at the top generatrix is larger than that at the bottom generatrix. At sections $d$ and $e$, the density is reduced observably and the large density variation regions disappear, hence the buoyancy effect here can be ignored. In Fig. 10(b), the significant buoyancy effect makes velocity profile distorted along the top half line at sections $b$ and $c$, where the "M" type profile appears meaning the lower shear stress. It may cause a very small turbulent kinetic energy, as shown in Fig. 10(d). Similar conclusions can be found in the previous literatures about water and carbon dioxide [8]. As heat transfer recovers to normal, velocity profile changes to the typical forced convective distribution at sections $d$ and $e$. It can be seen from Fig. 10(c), at section $b$, the fluid temperature increases drastically from $645 \mathrm{~K}$ at $y / R=0$ to $820 \mathrm{~K}$ at $y / R=1$, caused by the very low heat exchange between the flow core and the wall. At sections $d$ and $e$, the fluid temperature is above the pseudo-critical temperature, hence the variation of thermophysical property is diminished; meanwhile, turbulent kinetic energy is significantly increased, leading to heat transfer recovery.

As mentioned earlier, buoyancy effect may generate the secondary flow. Fig. 11 gives the secondary flow maps at five different cross sections in $2 \mathrm{~mm}$ diameter tube. The heated wall 
decreases the density, and the influence of buoyancy makes light fluid near the wall flow upward. Due to the blockage effect at the top wall, heavy fluid descends along the vertical axis, forming the vortex pair of the narrow long type, located slightly beneath the horizontal symmetry plane at section $a$. With the development of heat transfer deterioration, the buoyancy effect becomes more significant and the high density fluid is concentrated at the bottom half part of tube, leading to that the location of vortex pair moves vertically upward a bit and away from the wall at section $b$. At section $c$ where the density is reduced further, the vortex pair is pushed to the center of tube and the low temperature fluid at the bottom half part is driven to flow upward near the tube wall, causing the mixing between the high temperature and low temperature fluid, hence wall temperature descends. At section $d$, the vortex pair almost disappears meaning that buoyancy effect can be ignored at the time. At section $e$, although buoyancy effect is negligible, the density at the top half part is slightly lower than that at the bottom half part, also the higher axial velocity. In order to fill the vacancy at the top half part, another vortex pair arises with very tiny size.

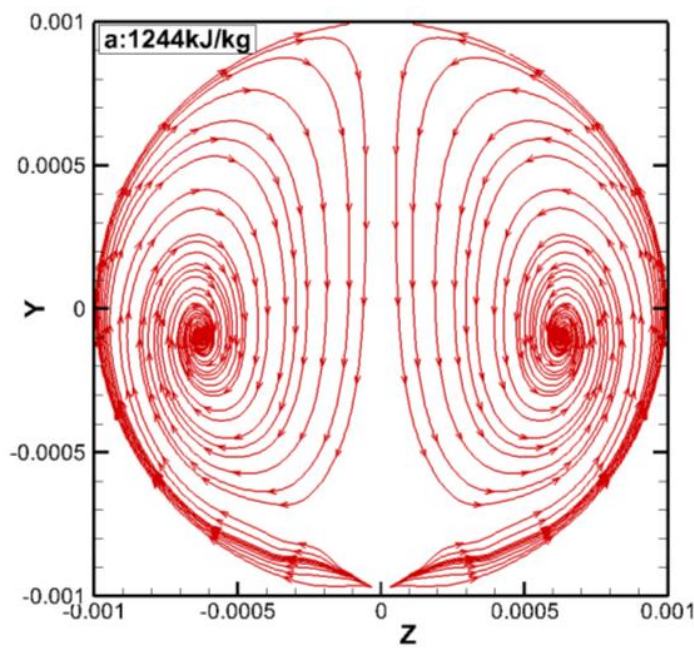

(a) section $a$

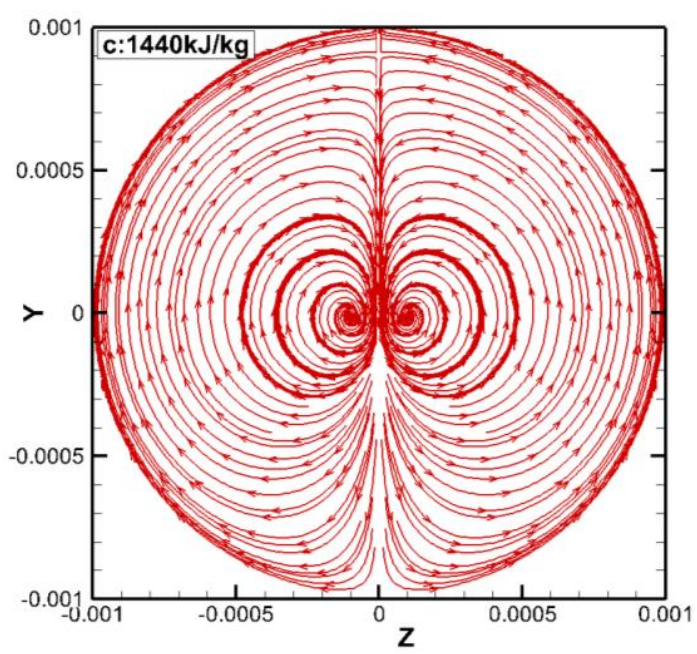

(c) section $c$

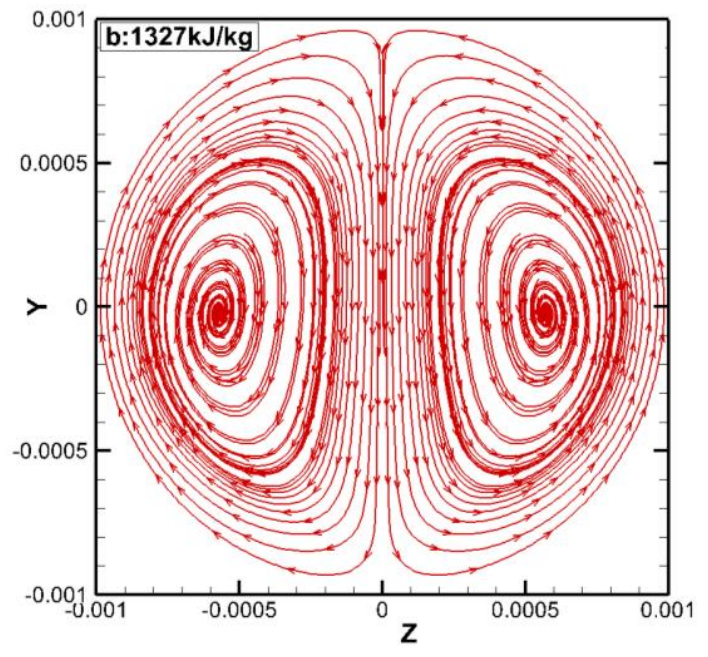

(b) section $b$

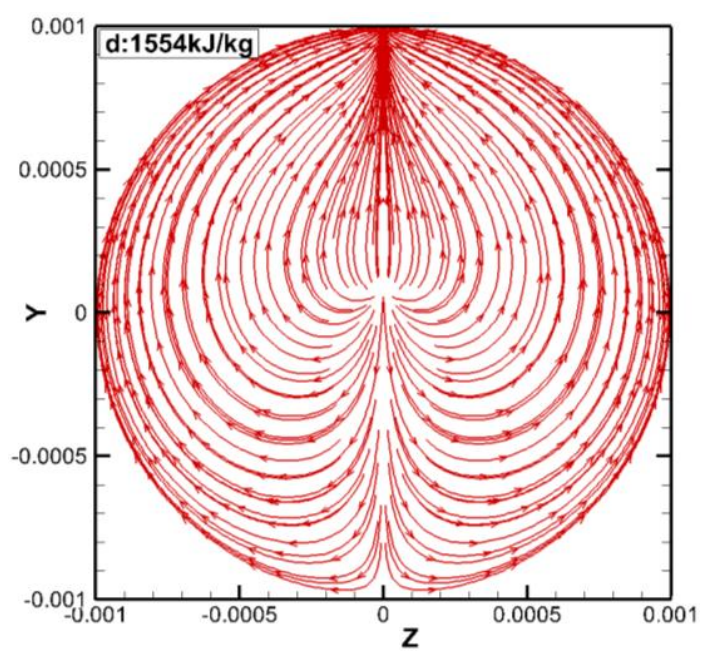

(d) section $d$ 


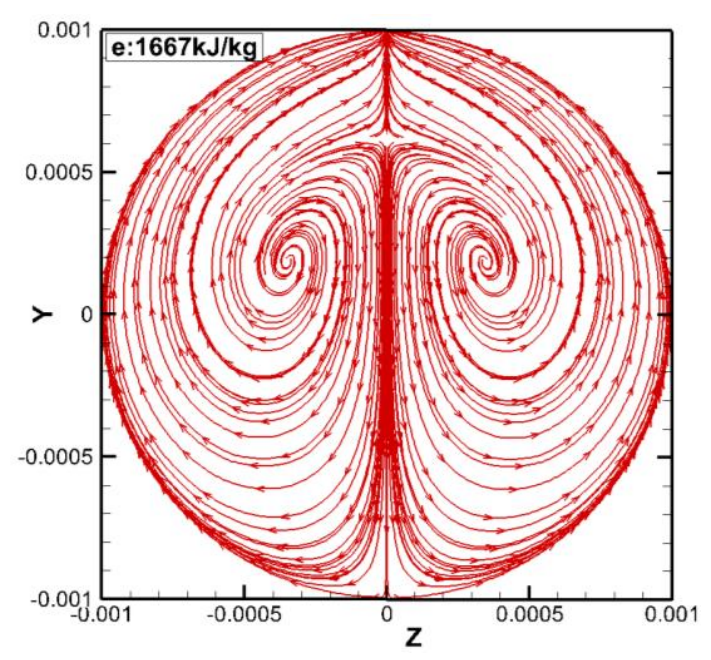

(e) section $e$

Fig. 11. Secondary flow maps at five cross sections in $2 \mathrm{~mm}$ tube.

In order to intuitively investigate the buoyancy effect on heat transfer characteristics in the horizontal tube, the variations of temperature with axial relative distance $x / d$ with and without buoyancy effect are presented in Fig. 12. Here NG means no gravity while MC means mix convection.

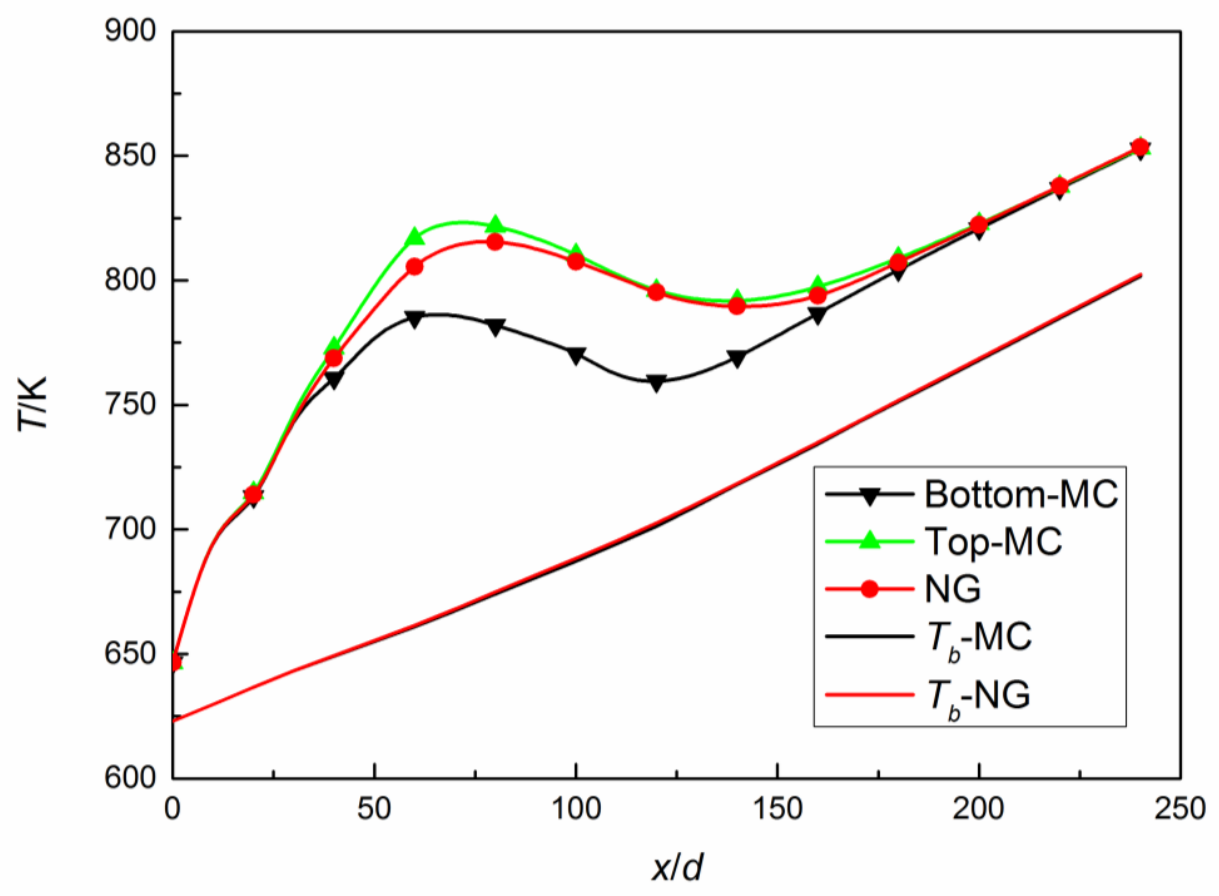

Fig. 12. Variations of temperature with and without buoyancy effect in $2 \mathrm{~mm}$ tube.

Compared with the temperature without buoyancy effect, it is noted from Fig. 12 that the buoyancy effect makes wall temperature at the top generatrix ascend, whereas wall temperature at the bottom generatrix descend more obviously. Whether the buoyancy effect is considered or not, heat transfer deterioration occurs, which may be caused by the thermal-induced acceleration effect due to the drastic change of density. The high temperature near the wall leads to the " $\mathrm{M}$ " type 
velocity profiles at $1330 \mathrm{~kJ} / \mathrm{kg}$ where wall temperature peak occurs (Fig. 13). Fig. 14 shows the secondary flow maps at $1327 \mathrm{~kJ} / \mathrm{kg}$ (heat transfer deterioration peak) and $1667 \mathrm{~kJ} / \mathrm{kg}$ (heat transfer recovery) without the buoyancy effect. There is no vortex appearing under the forced convection condition, so wall temperature becomes uniform along the circumference.

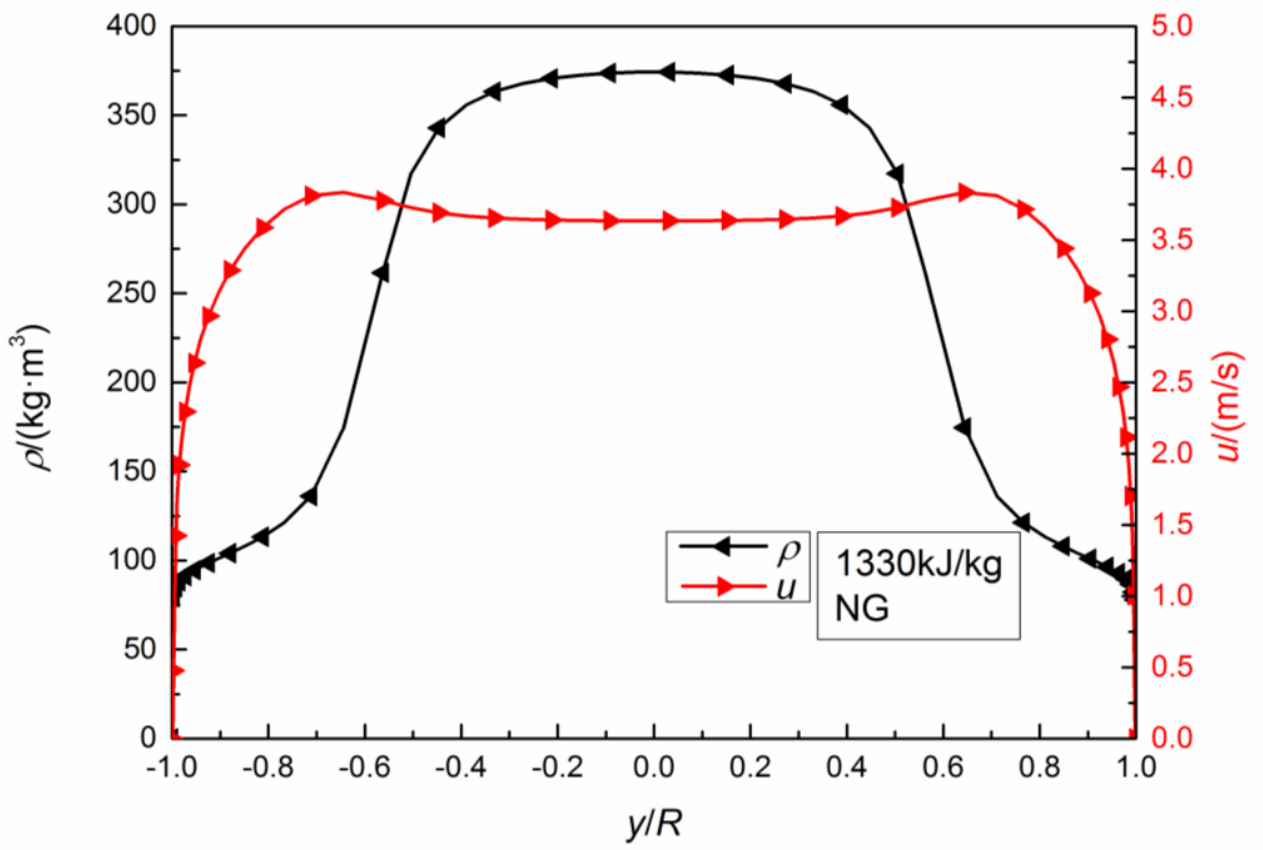

Fig. 13. Radial distribution of density and axial velocity at $1330 \mathrm{KJ} / \mathrm{kg}$ without buoyancy effect.

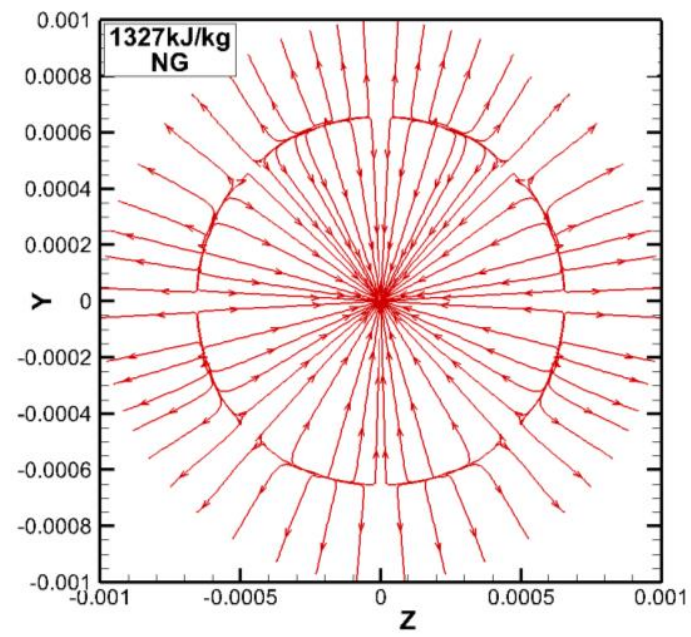

(a) $1327 \mathrm{~kJ} / \mathrm{kg}$

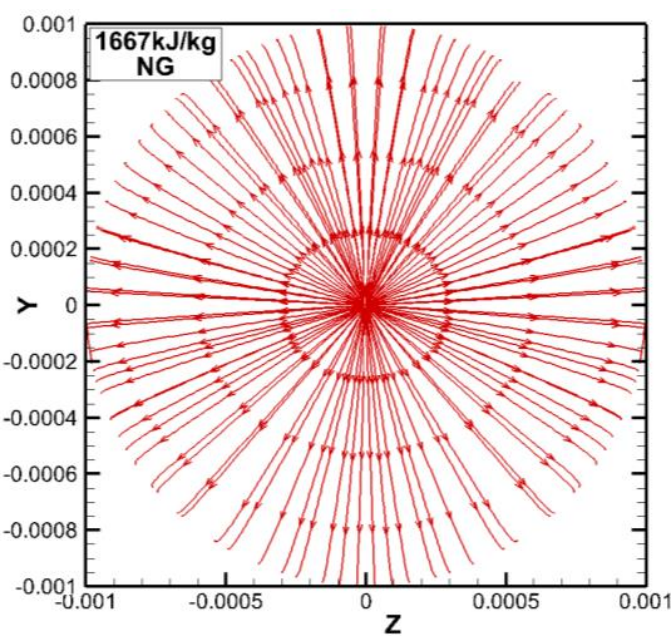

(b) $1667 \mathrm{~kJ} / \mathrm{kg}$

Fig. 14. Secondary flow maps at $1327 \mathrm{~kJ} / \mathrm{kg}$ and $1667 \mathrm{~kJ} / \mathrm{kg}$ without the buoyancy effect.

\subsection{Diameter effect on the heat transfer in the horizontal tube}

As the heat flux to mass flux ratio alters, various heat transfer modes under supercritical pressure can be observed, including heat transfer enhancement, heat transfer deterioration and normal heat transfer. Table 7 gives the computational conditions in the current section. 
Table 7 Computational conditions.

\begin{tabular}{ccccc}
\hline Case & $P / \mathrm{MPa}$ & $T_{\text {in }} / \mathrm{K}$ & $q / G /(\mathrm{kJ} / \mathrm{kg})$ & Model \\
\hline Case 1 & 3 & 623 & 0.71 & No. 1 \\
Case 2 & 3 & 623 & 0.71 & No. 2 \\
Case 3 & 3 & 623 & 0.71 & No. 3 \\
Case 4 & 3 & 623 & 0.71 & No. 4 \\
Case 5 & 3 & 623 & 0.71 & No. 5 \\
Case 6 & 3 & 623 & 0.25 & No. 1 \\
Case 7 & 3 & 623 & 0.25 & No. 2 \\
Case 8 & 3 & 623 & 0.25 & No. 3 \\
Case 9 & 3 & 623 & 0.25 & No. 4 \\
Case 10 & 3 & 623 & 0.25 & No. 5 \\
\hline
\end{tabular}

4.2.1 High heat flux to mass flux ratio

In comparison to low heat flux to mass flux ratio, the high value is likely to prompt the occurrence of heat transfer deterioration. Fig. 15 gives the variations of wall temperature with the fluid bulk enthalpy for cases 1-5. At the top generatrix, with the increases of diameter, the value of wall temperature peak increases, and the location of peak moves upstream meaning more serious heat transfer deterioration. At the bottom generatrix, the diameter has relatively little effect on wall temperature. Only when diameter ranges from $2 \mathrm{~mm}$ to $4 \mathrm{~mm}$, wall temperature has distinct change in the range of heat transfer deterioration. When tube diameter increases from $6 \mathrm{~mm}$ to $10 \mathrm{~mm}$, wall temperature almost keeps unchanged below $1400 \mathrm{~kJ} / \mathrm{kg}$. In heat transfer recovery region, wall temperature increases with increasing diameter at both the top and bottom generatrices.

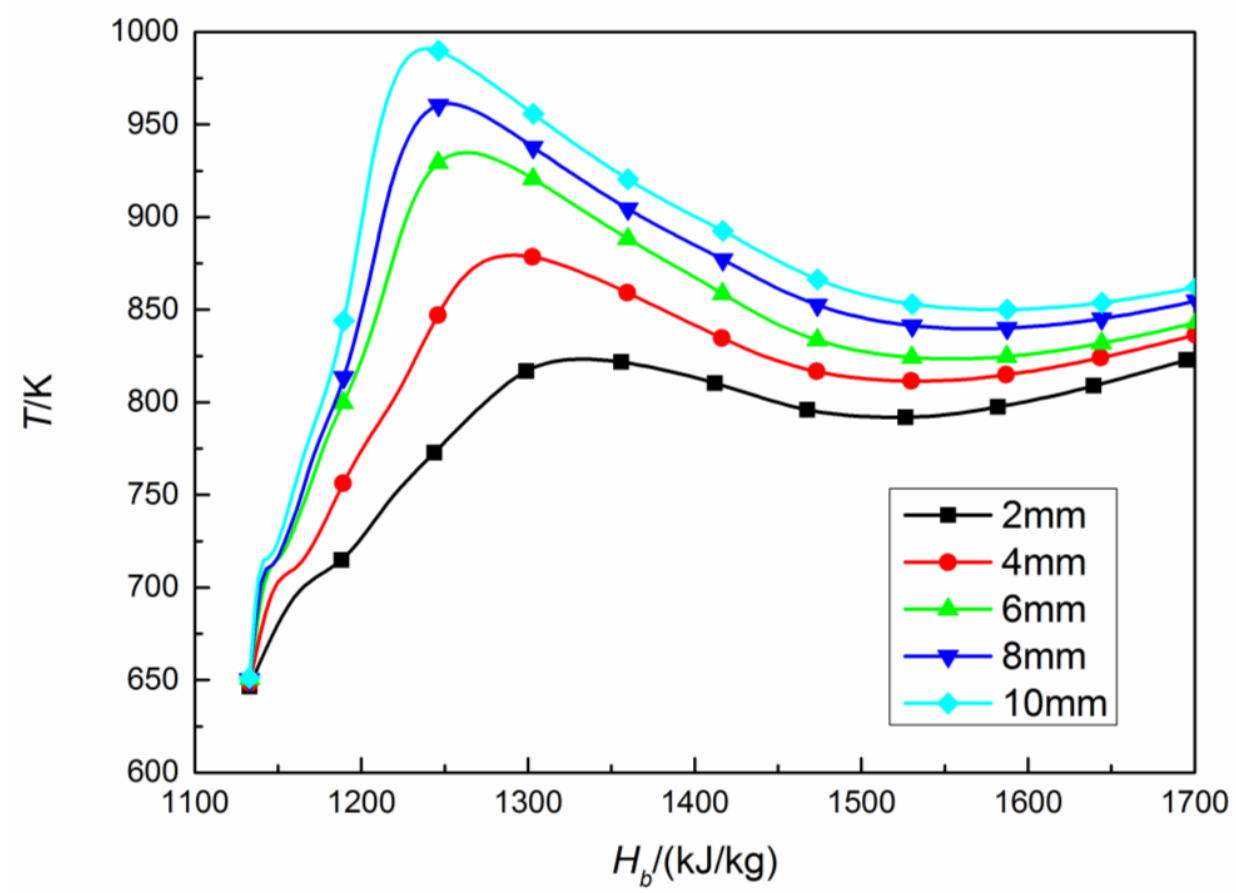

(a) top generatrix 


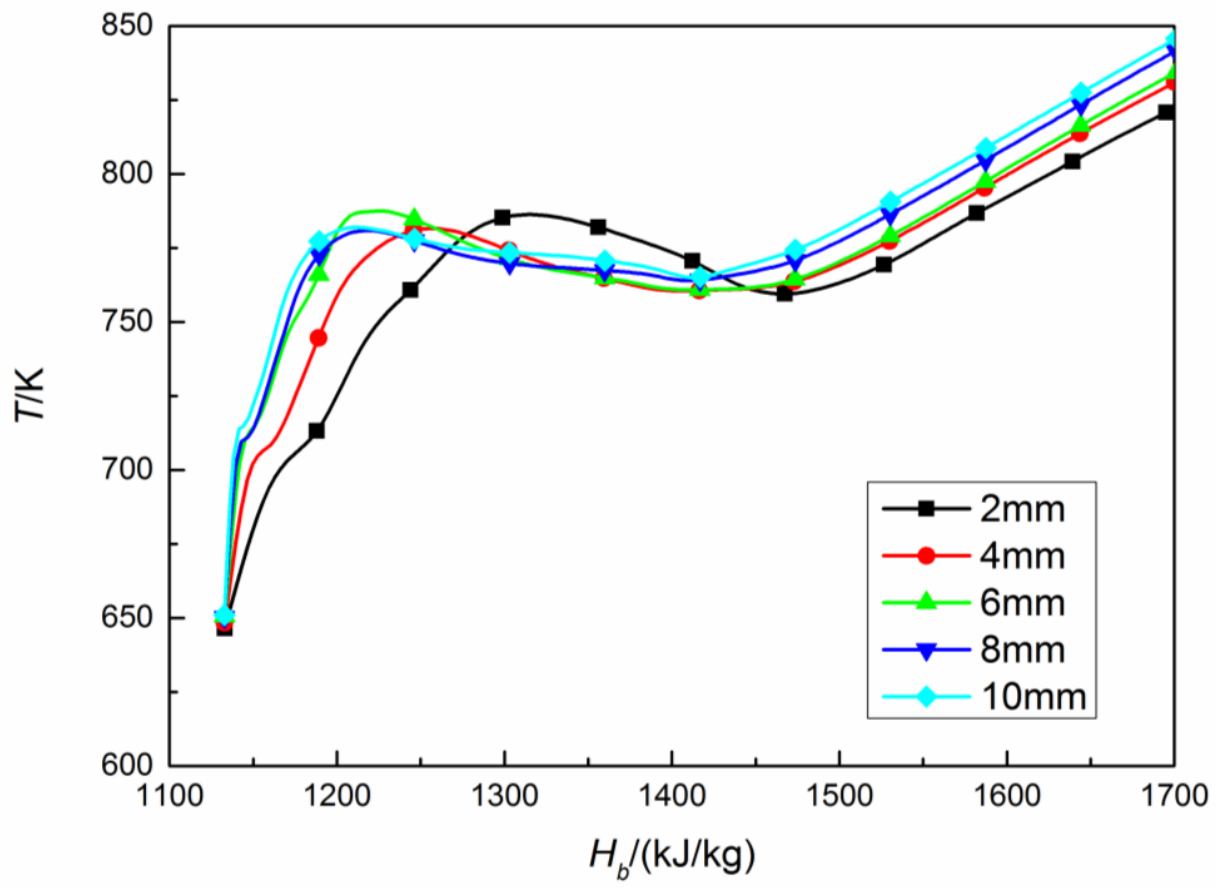

(b) bottom generatrix

Fig. 15. Variations of wall temperature with the fluid bulk enthalpy for cases 1-5.

As shown in Fig. 12, the buoyancy has more obvious effect on wall temperature at the bottom generatrix than that at the top generatrix in $2 \mathrm{~mm}$ tube. For the comparison study, the variations of temperature with axial relative distance $x / d$ with and without buoyancy effect in $10 \mathrm{~mm}$ tube are shown in Fig. 16.

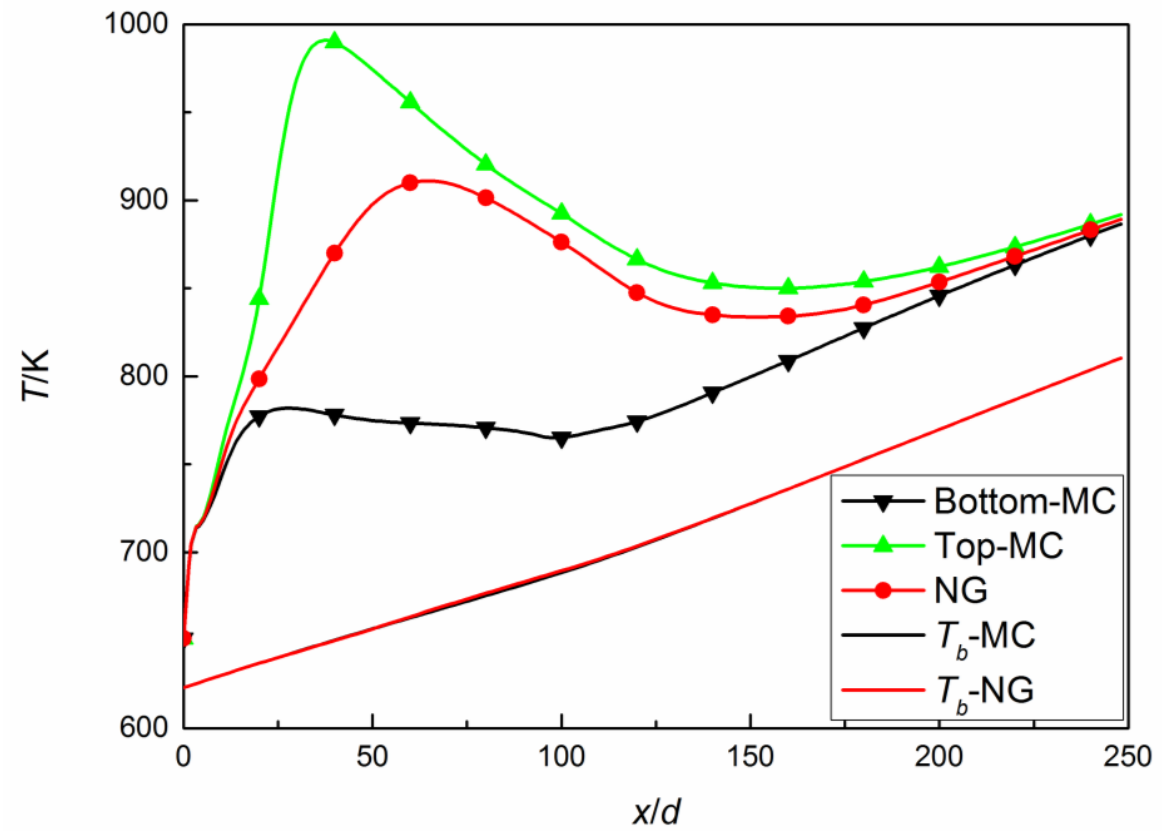

Fig. 16. Variations of temperature with and without buoyancy effect in $10 \mathrm{~mm}$ tube.

It can be seen that the buoyancy effect makes heat transfer at the bottom generatrix enhanced, 
compared with the forced convective heat transfer where buoyancy effect is removed, and heat transfer at the top generatrix deteriorated. $10 \mathrm{~mm}$ tube has similar tendency with $2 \mathrm{~mm}$ tube, that is, the obvious buoyancy effect at the bottom generatrix than that at the top generatrix. In regard to the diameter effect on heat transfer deterioration when buoyancy effect is removed, our previous article [41] gave the detailed study and found that, following the peak of specific heat, it abruptly decreases and then makes the reduced heat absorption capacity of the fluid, leading to heat transfer deterioration. With diameter increasing, heat transfer deterioration aggravates in NG case.

To quantitatively obtain the buoyancy and thermal-induced acceleration effect on heat transfer, the buoyancy parameter $B o^{*}[42]$ and thermal-induced acceleration parameter $K_{v}$ [43] are computed as below:

$$
\begin{gathered}
B o^{*}=G r^{*} / \operatorname{Re}^{3.425} \operatorname{Pr}^{0.8} \\
K_{v}=\frac{v_{b}}{u_{b}^{2}} \frac{d u_{b}}{d x}=\frac{4 q_{w} d \beta}{\operatorname{Re}^{2} \mu_{b} c_{p}} \\
G r^{*}=\frac{\beta g d^{4} q_{w}}{\lambda_{b} v_{b}^{2}} \\
\operatorname{Re}=\frac{\rho_{b} u_{b} d}{\mu_{b}} \\
\operatorname{Pr}=\frac{\mu_{b} c_{p}}{\lambda_{b}}
\end{gathered}
$$

Fig. 17 shows the variations of $B o^{*}$ and $K_{v}$ based on the fluid bulk temperature with fluid bulk enthalpy for cases 1-5.

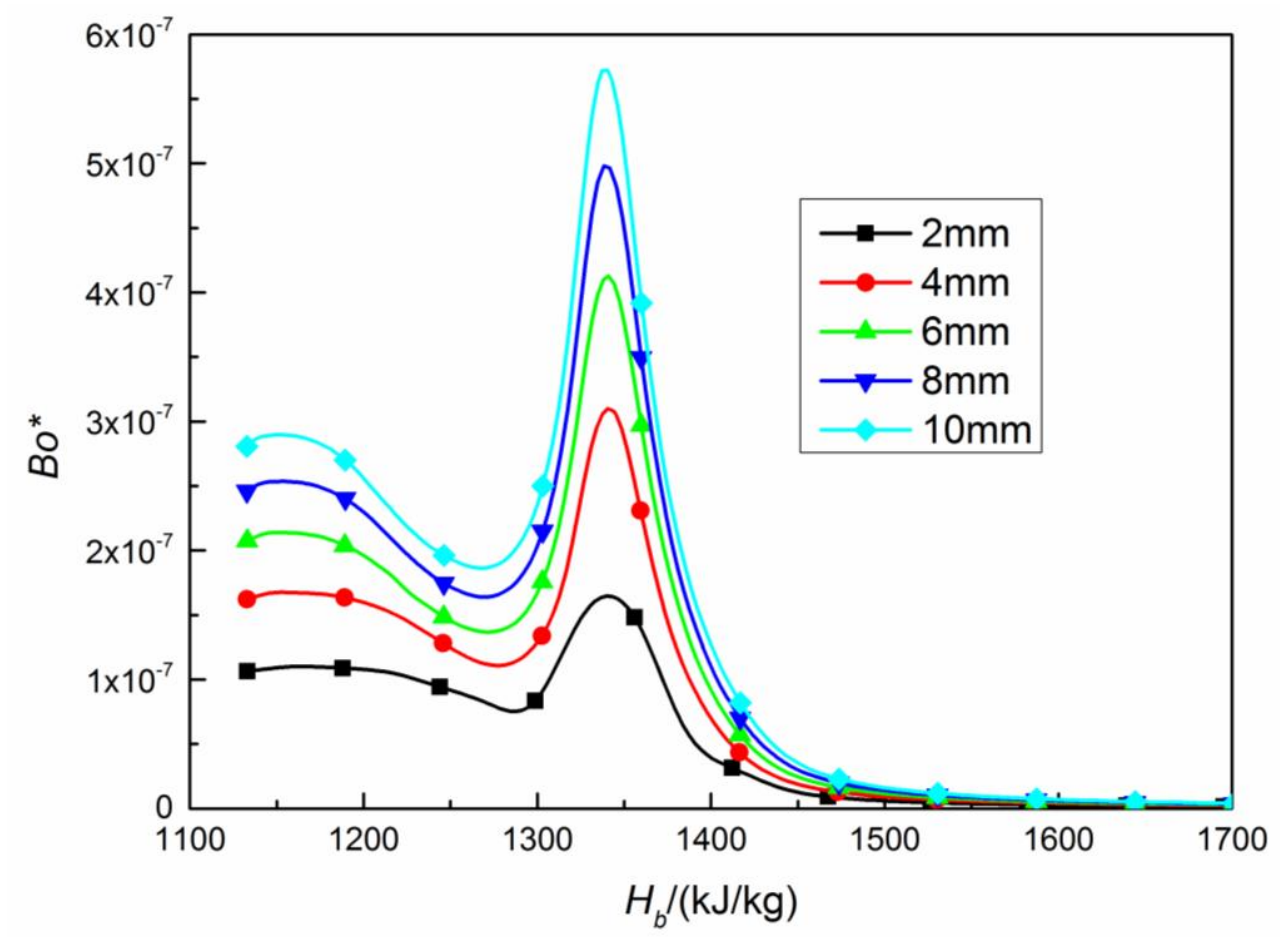




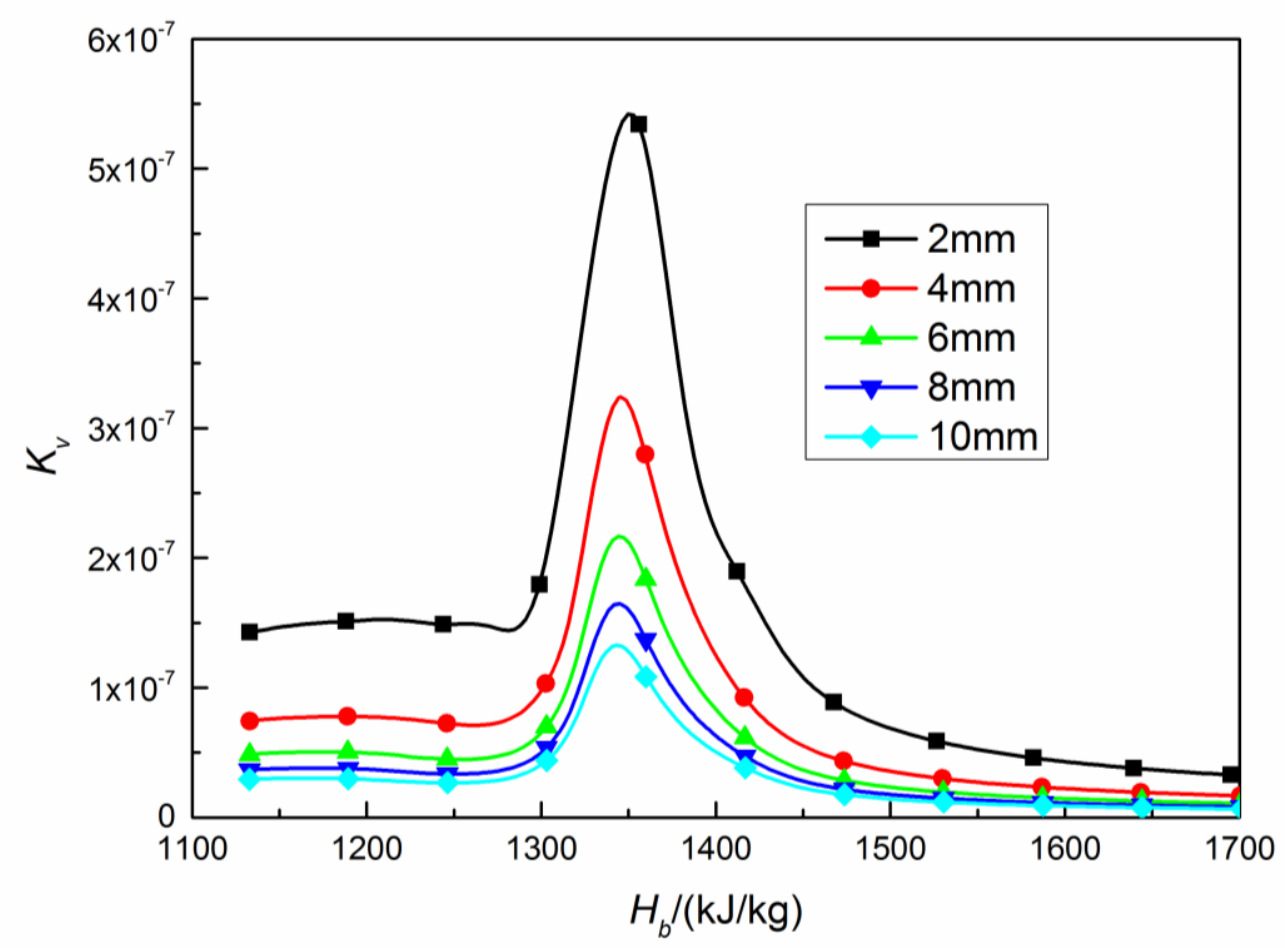

(b) $K_{v}$

Fig. 17. Variations of $B o^{*}$ and $K_{v}$ with the fluid bulk enthalpy for cases 1-5.

Seen from Fig. 17, as the increase of bulk enthalpy, the non-dimensional number $B o^{*}$ firstly slightly decreases until it reaches to a local minimum near $1300 \mathrm{~kJ} / \mathrm{kg}$, then abruptly ascends to the global maximum in the vicinity of $1350 \mathrm{~kJ} / \mathrm{kg}$ followed by the rapid declination towards to zero. When it comes to $K_{v}$, there is similar feature, but with the increase of diameter, $B O *$ increases and $K_{v}$ decreases, and the changing rates of both $\mathrm{Bo}^{*}$ and $K_{v}$ decrease. Larger buoyancy effect leads to almost unchanged temperature at the bottom generatrix in the larger diameter tube. The drastic change of thermophysical properties makes wall temperature at the top generatrix increase with the increasing diameter, with the thermal spike ahead of $B o^{*}$ and $K_{v}$ peak locations.

McEligot et al. [44] argued that the buoyancy effect should be considered when $B o^{*}>6 \times 10^{-7}$ for supercritical air flow, and the values of buoyancy effect parameters for all of the runs are lower than the proposed standard. However, significant buoyancy effect occurring in the current paper means that such a widely used criterion might not apply to the present study. In order to determine the onset of buoyancy effect influencing heat transfer in the current study, the curves of variations of wall temperature for different generatrices are compared with each other to find the range of the fluid bulk enthalpy corresponding to the divergence of different curves caused by buoyancy effect. Referring to Fig. 17a in which $B o^{*}$ varies with the fluid bulk enthalpy, it has been concluded that the threshold value of $B o^{*}$, whether buoyancy effect should be considered or not, is about less than $2.5 \times 10^{-8}$. Due to that the obvious buoyancy effect occurs in all the high heat flux to mass ratio cases, it's not easy to determine the accurate threshold value. In low heat flux to mass flux ratio cases, the further discussion about the threshold value of $B o^{*}$ will be given in the next section.

In the experiments of supercritical carbon dioxide [43], $K_{v}>3 \times 10^{-6}$ is proposed as a critical 
criterion to determine the thermal acceleration effect on heat transfer deterioration. The thermal-induced acceleration effect is caused by the volume expansion due to the variation of density at supercritical pressure. As mentioned earlier, the abrupt decrease of specific heat after the pseudo-critical point may lead to heat transfer deterioration. For all the cases, the value of thermal acceleration parameter is less than the proposed standard, however, it can be concluded from the results from NG cases in Figs. 12 and 16 that the combined action of thermal acceleration and specific heat has non-ignorable influence on heat transfer deterioration.

\subsubsection{Low heat flux to mass flux ratio}

Fig. 18 depicts the variations of wall temperature with the fluid bulk enthalpy for cases 6-10. Compared with cases 1-5, cases 6-10 have lower heat flux to mass flux ratio, leading to lower wall temperature with the maximum temperature not exceeding $760 \mathrm{~K}$. It can be seen that as diameter ranges from 2 to $4 \mathrm{~mm}$, heat transfer enhancement at the top generatrix appears at $1350 \mathrm{~kJ} / \mathrm{kg}$ where heat transfer deterioration occurs in the larger diameter tube. At the bottom generatrix, there is no obvious thermal peak in any tubes, and heat transfer enhancement occurs near $1350 \mathrm{~kJ} / \mathrm{kg}$, corresponding to $667 \mathrm{~K}$ near pseudo-critical temperature. At both top and bottom generatrices, the increasing diameter contributes to the increasing wall temperature.

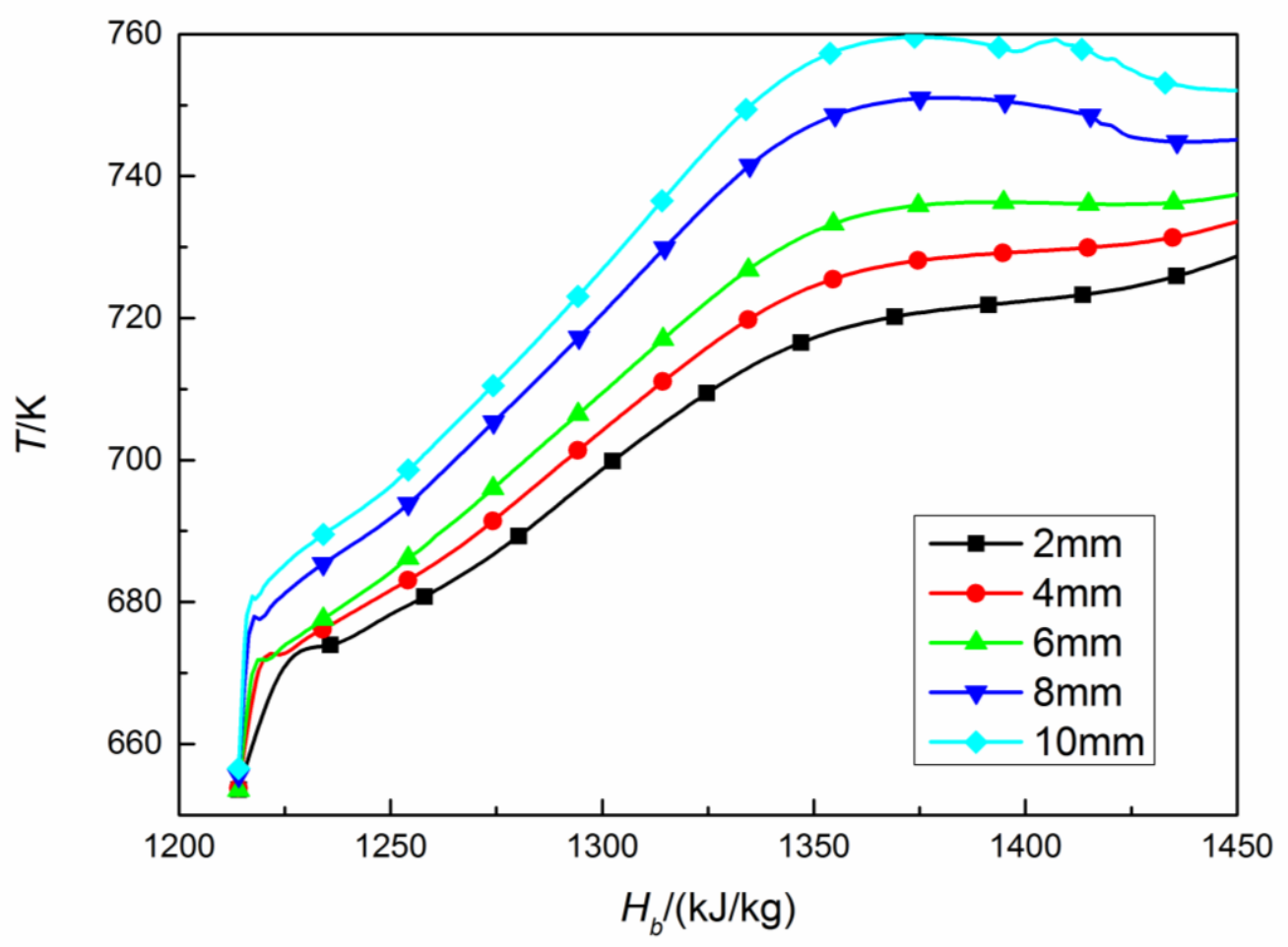

(a) top generatrix 


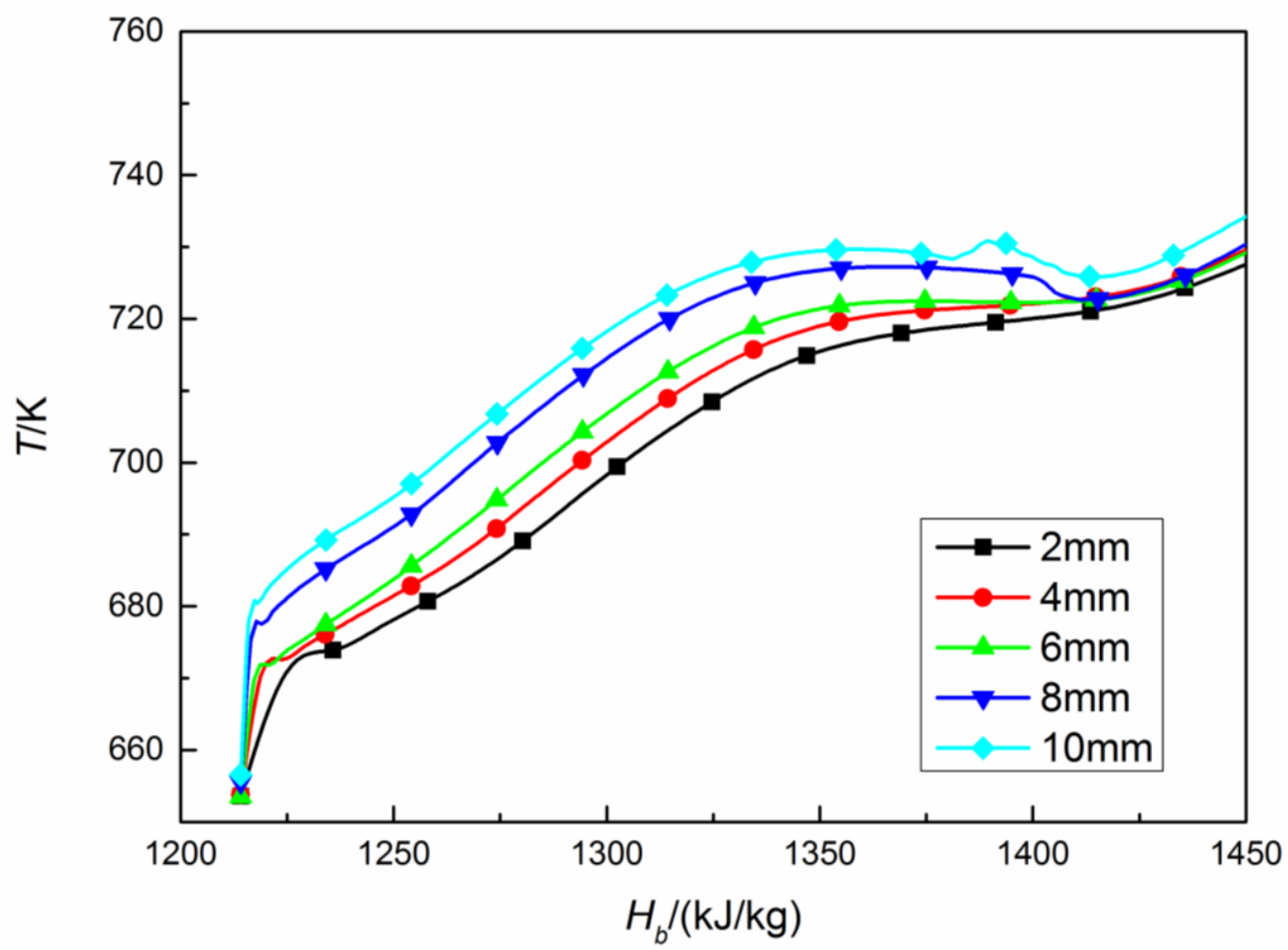

(b) bottom generatrix

Fig. 18. Variations of wall temperature with the fluid bulk enthalpy for cases 6-10.

Fig. 19 gives the variations of $B o^{*}$ and $K_{v}$ based on the fluid bulk temperature with the fluid bulk enthalpy for cases 6-10. By contrasting the temperature at the top generatrix to that at the bottom generatrix, it is concluded from Fig. 19 that the threshold value of buoyancy effect parameter $B o^{*}$ is near $1.0 \times 10^{-8}$. For all the cases in the current study, when $B o^{*}$ is less than the threshold value, buoyancy effect on supercritical heat transfer of hydrocarbon fuel could be ignored; when $B O^{*}$ is greater than the threshold value, the buoyancy has significant effect on supercritical heat transfer. In the public literature, the criterion of buoyancy effect on heat transfer deterioration has been given by Liu et al. [39] in their studies of n-decane flowing in the uniformly heated round tube at supercritical pressure. They thought as $B o^{*}>2 \times 10^{-7}$, buoyancy had significant influence on convection heat transfer. The different criterions for $B o^{*}$ might be caused by different working fluids which have different variation laws of thermophysical properties. Hydrocarbon fuel consists of a variety of hydrocarbons and has the potential chemical reaction at high temperature, which is very different from the pure substance.

The thermal-induced acceleration parameter $K_{v}$ in all the low heat flux to mass flux ratio cases is less than the proposed standard [43], and heat transfer remains enhanced due to the peculiar alteration of thermophysical properties, confirming the tiny effect of thermal acceleration on heat transfer deterioration. With the increase of temperature, specific heat undergoes a peak in the vicinity of $1350 \mathrm{~kJ} / \mathrm{kg}$, leading to a high heat absorption capacity, meanwhile, there is a small temperature difference between the wall and the flow core, hence a small thermophysical properties difference, making the heat from the high temperature wall transfer smoothly into the low temperature fluid. It is believed that the variation of specific heat plays a more important role in supercritical heat transfer where buoyancy effect is very tiny, compared with the variation of 
density.

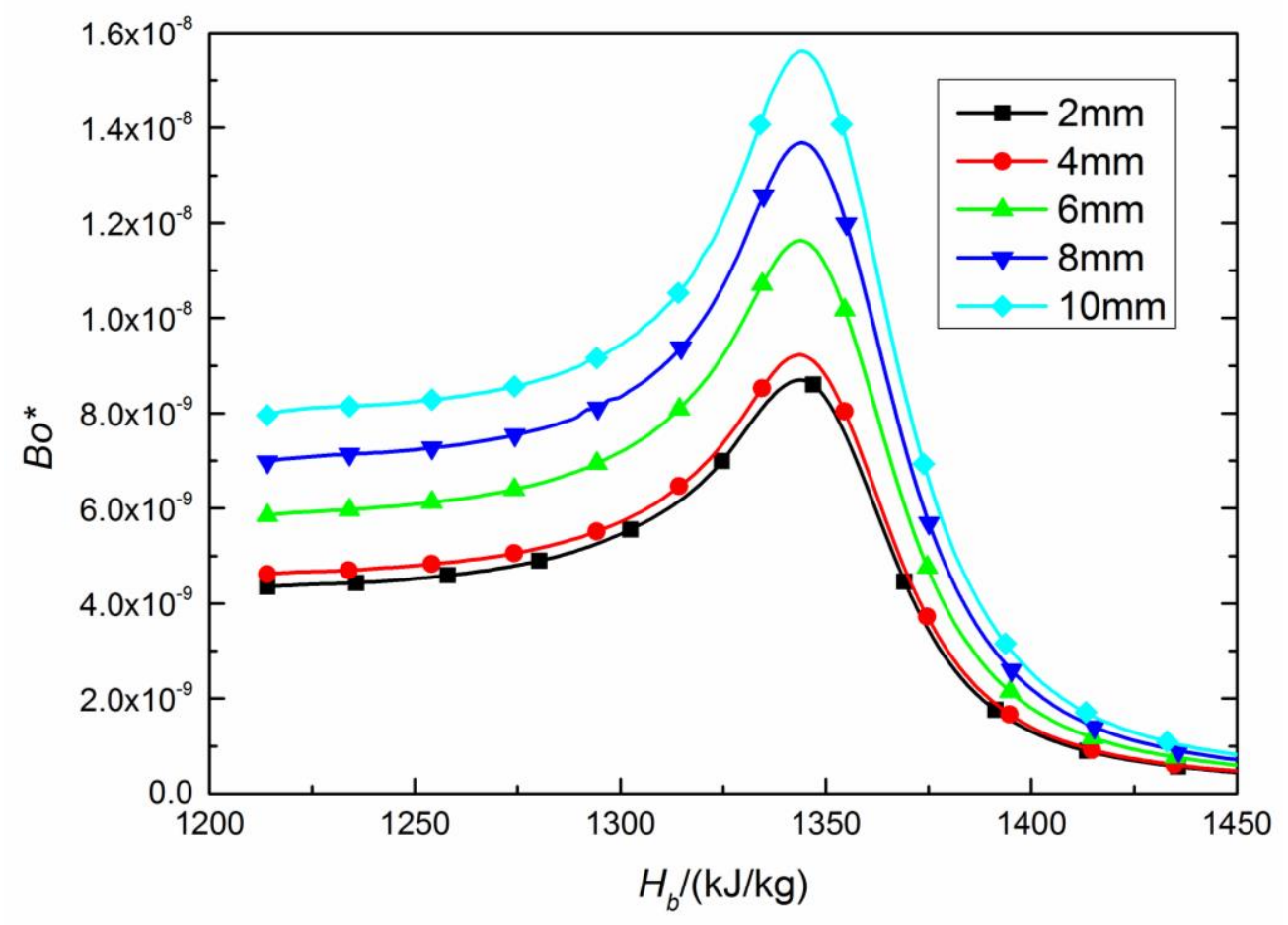

(a) $B o^{*}$

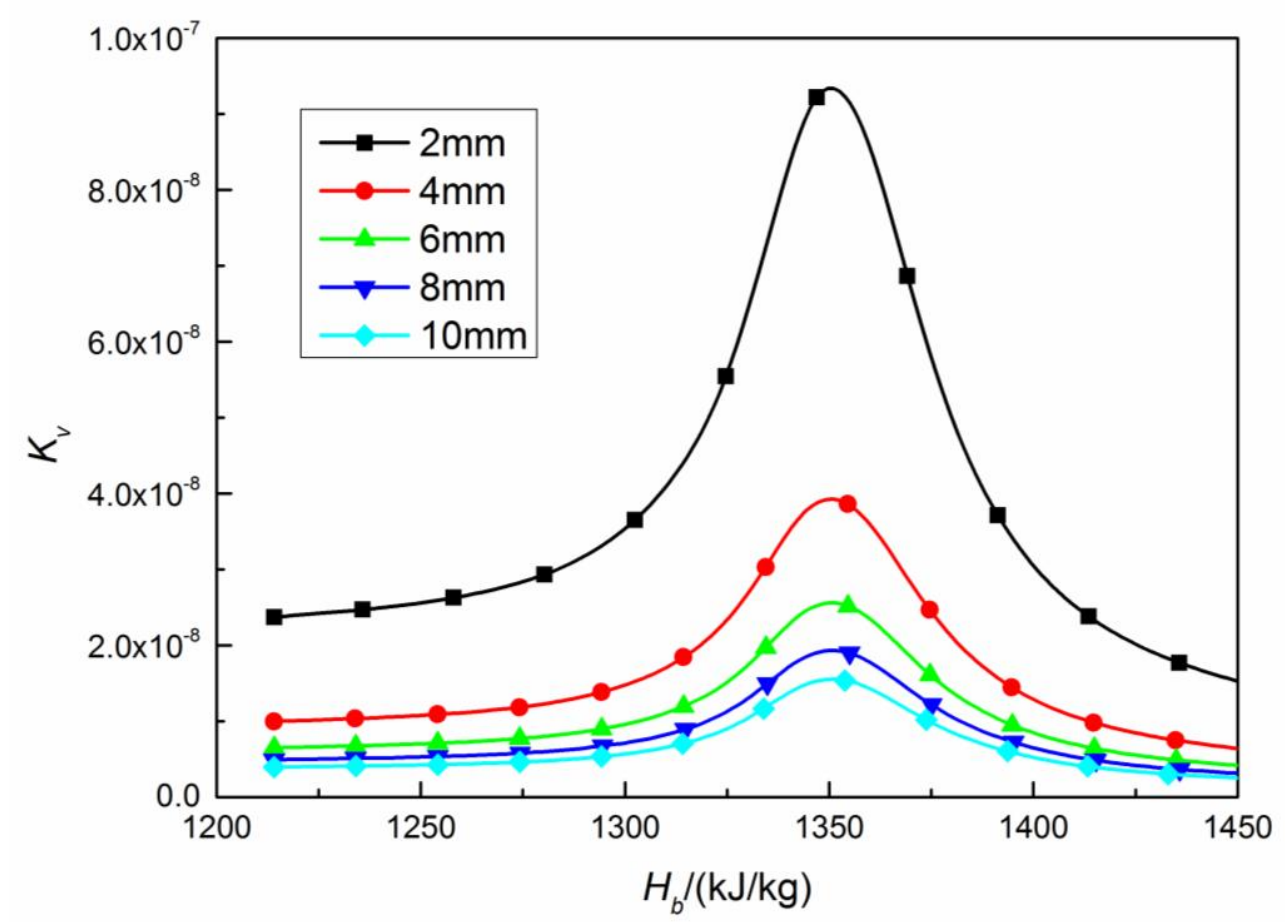

(b) $K_{v}$

Fig. 19. Variations of $B o^{*}$ and $K_{v}$ with the fluid bulk enthalpy for cases 6-10.

\subsection{Safety analysis}

As shown in the above sections, it is recognized that the diameter has significant contribution 
to the increase of wall temperature and can also make the conversion from heat transfer enhancement to heat transfer deterioration. In the large diameter tube, more obvious temperature non-uniformity in circumference direction has been detected which may lead to the great thermal stress, therefore the top surface in the horizontal tube is suggested to keep away from high temperature heat source. Based on the current analysis, small diameter tube would be a good choice to have a superior heat exchange capability provided that heat flux to mass flux ratio is not high.

\section{Conclusions}

In the current study, a method for computing the thermophysical properties of hydrocarbon fuel RP-3 at supercritical pressure has been proposed and a four-species surrogate model and a ten-species surrogate model of RP-3 have been assessed using the proposed method against the published data. Numerical scheme of supercritical heat transfer by CFD method embedded with LS low-Reynolds number turbulence model has been established and verified by comparing the computed results with the experimental data. Numerical simulation of supercritical heat transfer of RP-3 flowing in the horizontal uniformly heated tubes with various diameters has been carried out by the established numerical scheme. The heat transfer characteristics in the horizontal tube were analyzed by discussing the distribution of heat transfer data and detailed velocity fields. At high and low heat flux to mass flux ratios, the diameter effect on heat transfer in the horizontal tubes has been investigated in a systemic manner. The buoyancy effect and thermal-induced acceleration effect have been studied by proposing the critical criterion. Finally, the safety analysis has been given based on the discussion in the current paper. The main conclusions are shown below:

(1) Compared with the ten-species surrogate model of RP-3, a four-species model with the proposed thermophysical property algorithm based on the extended corresponding state principle can give a better prediction for the measured values of four thermophysical parameters.

(2) At supercritical pressure, there is obvious temperature non-uniformity along the circumference direction in the horizontal flow, with higher temperature and lower heat transfer coefficient at the top generatrix than those at the bottom generatrix caused by the buoyancy effect. As the pseudo-critical temperature is below the inner wall temperature and above the fluid bulk temperature, heat transfer deterioration occurs. As the fluid bulk temperature starts to exceed the pseudo-critical temperature, heat transfer begins to recover.

(3) The drastic change of thermophysical properties near pseudo-critical temperature leads to the unsymmetrical distribution of quantities profiles and then the occurrence of heat transfer deterioration. " $\mathrm{M}$ " type velocity profile is an important characteristic to determine the occurrence of heat transfer deterioration whether the buoyancy effect is considered or not.

(4) As heat transfer deterioration occurs, there is a pair of vortex making light fluid which has low heat absorption capability stay at the top half part. The evolution process of the pattern of vortex in the cross section reflects the formation, growth and decline of heat transfer deterioration.

(5) At high heat flux to mass flux ratio, as tube diameter increases from $2 \mathrm{~mm}$ to $10 \mathrm{~mm}$, wall temperature at the top generatrix increases and heat transfer deterioration is aggravated markedly. With the increase of diameter, buoyancy effect becomes more obvious and thermal-induced acceleration effect is reduced. The buoyancy effect makes temperature at the 
bottom generatrix decrease and temperature at the top generatrix increase but the effect at the bottom generatrix is more significant.

(6) At low heat flux to mass flux ratio, heat transfer enhancement occurs at both top and bottom generatrices near pseudo-critical temperature. In the circumstances, buoyancy effect and thermal-induced acceleration effect can be ignored compared with the drastic change of thermophysical property which leads to enhanced heat transfer.

(7) As the increase of bulk enthalpy, the buoyancy effect parameter $B o^{*}$ ascends to the global maximum followed by the rapid declination towards to zero. There is similar feature with the thermal acceleration parameter $K_{v}$. The critical criterion of $B o^{*}$ and $K_{v}$ obtained by the previous study on pure substance might not be suitable to the current study on RP-3. A threshold value for $B o^{*}$ has been obtained as $1.0 \times 10^{-8}$, above which buoyancy influences heat transfer obviously.

By comparing the critical criterion in the current study with similar studies in literatures, the characteristic of buoyancy effect for RP-3 is different from that for pure substance, supplementing the buoyancy theory in supercritical heat transfer. More importantly, the law of heat transfer deterioration for supercritical hydrocarbon fuel in the horizontal tubes of various diameters has been grasped and it is very helpful in avoiding the occurrence of heat transfer deterioration when designing the regenerative cooling system of the Scramjet.

(8) For safety consideration, high temperature heat source should keep away from the top half part of horizontal tube. In the design of hydrocarbon fuel cooling system, small diameter tube is recommended to achieve a good heat exchange performance.

In the current study, the variation of density was not separated from the integral computation, so the critical threshold value of thermal acceleration effect parameter $K_{v}$ has been not obtained. In future work, each of four thermophysical property parameters needs to be studied separately by changing the thermophysical property model and the onset of heat transfer deterioration can be further study which is of great importance to avoid high wall temperature.

\section{Acknowledgement}

The authors gratefully acknowledge funding support from Program for National Natural Science Foundation of China (51406005).

\section{References}

[1] E.T. Curran, Scramjet engines: the first forty years, J. Propul. Power 17 (2001) 1138-1148.

[2] C.B. Zhang, Investigation of flow and heat transfer characteristics of hydrocarbon fuel at supercritical pressures, PhD Thesis, Beihang University, 2011.

[3] D.R. Sobei, L.J. Spadaccin, Thermal management for a Mach 5 cruise aircraft using endothermic fuel, J. Eng. Gas. Turbines Power-Trans. ASME 119 (1997) 344-351.

[4] B. Berche, M. Henkel, R. Kenna, Critical phenomena: 150 years since Cagniard de la Tour, Revista Brasileira De Ensino De Física 13 (2009) 2602.1-2602.4.

[5] J.D. Jackson. Fluid flow and convective heat transfer to fluids at supercritical pressure, Nucl. Eng. Des. 264 (2013) 24-40.

[6] Y. Chen, Y. Wang, Z. Bao, Q. Zhang, X.Y. Li, Numerical investigation of flow distribution and heat transfer of hydrocarbon fuel in regenerative cooling panel, Appl. Therm. Eng. 98 (2016) 628-635. 
[7] V.A. Kurganov, Y.A. Zeigarnik, I.V. Maslakova, Heat transfer and hydraulic resistance of supercritical-pressure coolants. Part I: Specifics of thermophysical properties of supercritical pressure fluids and turbulent heat transfer under heating conditions in round tubes (state of the art), Int. J. Heat Mass Transf. 55 (2012) 3061-3075.

[8] D. Huang, Z. Wu, B. Sunden, W. Li, A brief review on convection heat transfer of fluids at supercritical pressures in tubes and the recent progress, Appl. Energy 162 (2016) 494-505.

[9] N.T. ERao, A.N. Oumer, U.K. Jamaludin, State-of-the-art on flow and heat transfer characteristics of supercritical $\mathrm{CO}_{2}$, in various channels, J. Supercrit. Fluids 116 (2016) 132-147.

[10] K. Yamagata, K. Nishikawa, S. Hasegawa, T. Fujii, S. Yoshida, Forced convective heat transfer to supercritical water flowing in tubes, Int. J. Heat Mass Transf. 15 (1972) 2575-2593.

[11] B.S. Shiralkar, P. Griffith, Deterioration in heat transfer to fluids at supercritical pressure and high heat fluxes, J. Heat Transf.-Trans. ASME (1969) 27-36.

[12] X.L. Lei, H.X. Li, W.Q. Zhang, N.T. Dinh, Y.M. Guo, S.Q. Yu, Experimental study on the difference of heat transfer characteristics between vertical and horizontal flows of supercritical pressure water, Appl. Therm. Eng. 113 (2017) 609-620.

[13] X.L. Lei, H.X. Li, Y.F. Zhang, W.Q. Zhang, Effect of buoyancy on the mechanism of heat transfer deterioration of supercritical water in horizontal tubes, J. Heat Transf.-Trans. ASME 135 (2013) 071703.

[14] Z.G. Gao, J.H. Bai, Numerical analysis on nonuniform heat transfer of supercritical pressure water in horizontal circular tube, Appl. Therm. Eng. 120 (2017) 10-18.

[15] Z. Shang, S. Chen, Numerical investigation of diameter effect on heat transfer of supercritical water flows in horizontal round tubes, Appl. Therm. Eng. 31 (2011) 573-581.

[16] S.Q. Yu, H.Q. Li, X.L. Lei, Y.C. Feng, Y.F. Zhang, H. He, T. Wang, Influence of buoyancy on heat transfer to water flowing in horizontal tubes under supercritical pressure, Appl. Therm. Eng. 59 (2013) 380-388.

[17] S. Yildiz, D.C. Groeneveld, Diameter effect on supercritical heat transfer, Int. Commun. Heat Mass Transf. 54 (2014) 27-32.

[18] Y. Guo, Q.C. Bi, Z.H. Liu, Z.Q. Yang, L. Jiang, Experimental investigation on thermal-hydraulic characteristics of endothermic hydrocarbon fuel in $1 \mathrm{~mm}$ and $2 \mathrm{~mm}$ diameter mini-channels, Appl. Therm. Eng. 122 (2017) 420-428.

[19] Z.B. Liu, Y.L. He, Y.F. Yang, J.Y. Fei, Experimental study on heat transfer and pressure drop of supercritical $\mathrm{CO}_{2}$, cooled in a large tube, Appl. Therm. Eng. 70 (2014) 307-315.

[20] L.F. Cabeza, A.D. Gracia, A.I. Fernández, M.M. Farid, Supercritical $\mathrm{CO}_{2}$ as heat transfer fluid: A review, Appl. Therm. Eng. 125 (2017) 799-810.

[21] J.D. Jackson, Models of heat transfer to fluids at supercritical pressure with influences of buoyancy and acceleration, Appl. Therm. Eng. 124 (2017) 1481-1491.

[22] M. Yang, Numerical study of the heat transfer to carbon dioxide in horizontal helically coiled tubes under supercritical pressure, Appl. Therm. Eng. 109 (2016) 685-696.

[23] T. Edwards, L.Q. Maurice, Surrogate Mixtures to Represent Complex Aviation and Rocket Fuels, J. Propul. Power 17 (2012) 461-466.

[24] P. Dagaut, M. Cathonnet, The ignition, oxidation, and combustion of kerosene: A review of experimental and kinetic modeling, Prog. Energy Combust. Sci. 32 (2006) 48-92.

[25] F.Q. Zhong, X.J. Fan, G. Yu, J.G. Li, C.J. Sung, Heat transfer of aviation kerosene at supercritical conditions, J. Thermophys. Heat Transf. 23 (2009) 543-550.

[26] B.A. Younglove, J.F. Ely, Thermophysical properties of fluids. II. Methane, ethane, propane, isobutane, and normal butane, J. Phys. Chem. Ref. Data 16 (1987) 577-798.

[27] J.F. Ely, H.J.M. Hanley, Prediction of transport properties. 1. Viscosity of fluids and mixtures, Ind. Eng. Chem. Fundamen. 20 (1981) 323-332.

[28] K. Lucas, Phase equilibria and fluid properties in the chemical industry, Frakfurt, Dechema, 1980.

[29] H.W. Deng, K. Zhu, G.Q. Xu, Z. Tao, C.B. Zhang, G.Z. Liu, Isobaric specific heat capacity measurement for kerosene RP-3 in the near-critical and supercritical regions, J. Chem. Eng. Data 57 (2011) 263-268.

[30] H.W. Deng, C.B. Zhang, G.Q. Xu, Z. Tao, B. Zhang, G.Z. Liu, Density measurements of endothermic hydrocarbon fuel at sub-and supercritical conditions, J. Chem. Eng. Data 56 (2011) 2980-2986.

[31] H.W. Deng, C.B. Zhang, G.Q. Xu, B. Zhang, Z. Tao, K. Zhu, Viscosity measurements of endothermic hydrocarbon fuel from (298 to 788$) \mathrm{K}$ under supercritical pressure conditions, J. Chem. Eng. Data 57 (2012) 358-365.

[32] H.W. Deng, C.B. Zhang, G.Q. Xu, Z. Tao, K. Zhu, Y.J. Wang, Visualization experiments of a 
specific fuel flow through quartz-glass tubes under both sub- and supercritical conditions, Chin. J. Aeronaut. 25 (2012) 372-380.

[33] Z.Q. Liu, J.H. Liang, Y, Pan, Numerical analysis of heat transfer deterioration of China RP-3 aviation kerosene in a circular tube at supercritical pressures, AIAA paper 2014-3358.

[34] M. Pizzarelli, F. Nasuti, R. Votta, F. Battista, Validation of Conjugate Heat Transfer Model for Rocket Cooling with Supercritical Methane, J. Propul. Power 32 (2016) 1-8.

[35] W.S. Kim, S. He, J.D. Jackson, Assessment by comparison with DNS data of turbulence models used in simulations of mixed convection, Int. J. Heat Mass Transf. 51 (2008) 1293-1312.

[36] Z. Tao, Z.Y. Cheng, J.Q. Zhu, H.W. Li, Effect of turbulence models on predicting convective heat transfer to hydrocarbon fuel at supercritical pressure, Chin. J. Aeronaut. 29 (2016) 1247-1261.

[37] B.E. Launder, B.I. Sharma, Application of the energy-dissipation model of turbulence to the calculation of flow near a spinning disc, Lett. Heat Mass Transf. 1 (1974) 131-137.

[38] Fluent 14.5 User Guide, Fluent Inc., Lebanon, USA, 2013.

[39] B. Liu, Y.H. Zhu, J.J. Yan, Y.T. Lei, B. Zhang, P.X. Jiang, Experimental investigation of convection heat transfer of n-decane at supercritical pressures in small vertical tubes, Int. J. Heat Mass Transf. 91 (2015) 734-746.

[40] Y.H. Zhu, B. Liu, P.X. Jiang, Experimental and numerical investigations on n-decane thermal cracking at supercritical pressures in a vertical tube, Energy Fuels 28 (2013) 466-474.

[41] Z.Y. Cheng, J.Q. Zhu, H.W. Li, Diameter effect on heat transfer deterioration of supercritical hydrocarbon fuel in vertical round tubes, Acta Aeronautica et Astronautica Sinica 37 (2016) 2941-2951.

[42] J.D. Jackson, W.B. Hall, Influence of buoyancy on heat transfer to fluids flowing in vertical tubes under turbulent conditions, Turbulent forced convection in channels and bundles 2 (1979) 613-640.

[43] D.M. McEligot, C.W. Coon, H.C. Perkins, Relaminarization in tubes, Int. J. Heat Fluid Flow 13 (1970) 431-433.

[44] D.M. McEligot, J.D. Jackson, “Deterioration” criteria for convective heat transfer in gas flow through non-circular ducts, Nucl. Eng. Des. 232 (2004) 327-333. 